\title{
Structural and mechanical characterisation of bridging veins: a review
}

\author{
Nele Famaey ${ }^{\mathrm{a}, *}$, Zhao Ying Cui ${ }^{\mathrm{a}, *}$, Grace Umuhire Musigazi ${ }^{\mathrm{b}}$, Jan Ivens ${ }^{\mathrm{c}}$, \\ Bart Depreitere $^{\mathrm{b}}$, Erik Verbeken ${ }^{\mathrm{d}}$, Jos Vander Sloten ${ }^{\mathrm{a}}$ \\ ${ }^{a}$ Biomechanics Section, KU Leuven, Belgium \\ ${ }^{b}$ Department of Neurosurgery, University Hospital Gasthuisberg, KU Leuven, Belgium \\ ${ }^{c}$ Composite Materials Group, Department of Metallurgy and Materials Engineering, $K U$ \\ Leuven, Belgium \\ ${ }^{d}$ Translational Cell $\& 3$ Tissue Research, KU Leuven, Belgium
}

\begin{abstract}
Bridging veins drain the venous blood from the cerebral cortex into the superior sagittal sinus (SSS) and doing so they bridge the subdural space. Despite their importance in head impact biomechanics, little is known about their properties with respect to histology, morphology and mechanical behaviour. Knowledge of these characteristics is essential for creating a biofidelic finite element model to study the biomechanics of head impact, ultimately leading to the improved design of protective devices by setting up tolerance criteria. This paper presents a comprehensive review of the state-of-the-art knowledge on bridging veins.

Tolerance criteria to prevent head injury through impact have been set by a number of research groups, either directly through impact experiments or by means of finite element (FE) simulations. Current state-of-the-art FE head models still lack a biofidelic representation of the bridging veins. To achieve this, a thorough insight into their nature and behaviour is required. Therefore, an overview of the general morphology and histology is provided here, showing the clearly heterogeneous nature of the bridging vein complex, with its three different layers and distinct morphological and histological changes at the region of outflow into the superior sagittal sinus. Apart from a complex morphology, bridging veins also exhibit complex mechanical
\end{abstract}

*Authors contributed equally

Correspondence to: nele.famaey@kuleuven.be, tel: +32 16328980

Preprint submitted to JMBBM

July 24, 2014 
behaviour, being nonlinear, viscoelastic and prone to damage. Existing material models capable of capturing these properties, as well as methods for experimental characterization, are discussed.

Future work required in bridging vein research is firstly to achieve consensus on aspects regarding morphology and histology, especially in the outflow cuff segment. Secondly, the advised material models need to be populated with realistic parameters through biaxial mechanical experiments adapted to the dimensions of the bridging vein samples. Finally, updating the existing finite element head models with these parameters will render them truly biofidelic, allowing the establishment of accurate tolerance criteria and, ultimately, better head protection devices.

Keywords: bridging veins, anatomy, mechanical properties, finite element modeling, tolerance criteria

\section{Introduction}

Haemorrhage inside the cranial cavity resulting in the formation of a haematoma is a well known cause of death and disability after head trauma. Depending on the site of haemorrhage, haematomas are classified as either extradural, subdural or intracerebral ${ }^{(1)}$. Acute subdural haematoma (ASDH) is a very common head lesion (the most frequent traumatic head injury in bicycle-related accidents ${ }^{(2)}$ ) with mortality rate ranging from $30 \%$

to $90 \%{ }^{(3,4)}$, making it a non-negligible pathology. Next to head contusion and laceration of the cerebral veins and arteries, a rupture of the bridging veins $(\mathrm{BV})$ is one the main reasons of $\mathrm{ASDH}$, accounting for one third of all the $\operatorname{cases}^{(3,5)}$.

Bridging veins are in charge of draining the blood from the cerebral cortex into the superior sagittal sinus (SSS). Despite the importance of the BVs in the etiology of ASDH, still little is known about their properties, with respect to histology, morphology and mechanical properties. Knowledge of these properties is necessary for creating a biofidelic finite element model ${ }^{(6,7)}$ which is used to study the biomechanics of head impact. This in turn will improve the design of protective devices by setting up tolerance criteria as well as benefit forensic science.

This paper presents an extensive overview of the state-of-the-art knowledge on bridging veins. First of all, tolerance criteria for bridging vein laceration are discussed, showing a lack of consensus and hence the pressing need 
for more biofidelic head models with accurate geometrical and mechanical representation of the bridging veins. Consequently, section 3 discusses current knowledge on the general structure of the bridging vein, covering gross anatomy, histology and characterisation techniques. Apart from a complex morphology, bridging veins also exhibit complex mechanical behaviour, being nonlinear, viscoelastic and prone to damage. These properties, as well as ways to mechanically characterize and mathematically describe them, are discussed in section 4. Next, state-of-the-art finite element head models are described with a focus on how each of these models deal with the bridging veins. Finally, a general discussion and suggestions for future work are provided.

\section{Tolerance criteria}

The maximum impact a head can bear without damage is represented by tolerance criteria. The most commonly used criterion is the head in-

jury criterion $(\mathrm{HIC})^{(8)}$, which is based on the translational acceleration of the head, disregarding rotational acceleration, impact force and directional dependency:

$$
H I C=\left\{\left(t_{2}-t_{1}\right)\left[\frac{1}{t_{2}-t_{1}} \int_{t_{1}}^{t_{2}} a(t) d t\right]^{2.5}\right\}_{\max },
$$

with $a(t)$ the resultant translational head acceleration and $t_{1}$ and $t_{2}$ the initial and final time of the interval selected to maximize the HIC value. However, Gennarelli et al. concluded that purely translational motions cannot induce ASDH and the importance of rotational acceleration for the rupture of bridging veins has been stated by multiple research groups ${ }^{(9,10,11,12,1,13)}$. Contrary to the HIC, the GAMBIT (Generalized Acceleration Model for Brain Injury Threshold) predicts head injury taking into account both translational and rotational acceleration ${ }^{(14)}$. The original form of the criterion is:

$$
G A M B I T=\left[\left(\frac{a(t)}{a_{c}}\right)^{n}+\left(\frac{\alpha(t)}{\alpha_{c}}\right)^{m}\right]^{\frac{1}{s}},
$$

with $a(t)$ and $\alpha(t)$ the instantaneous values of the translational and rotational acceleration, respectively. $a_{c}$ and $\alpha_{c}$ indicate the critical acceleration levels for a pure translational and a pure rotational pulse, respectively. $n, m$ 
and $s$ are empirical constants which are fitted to the available data. However the lack of pulse duration dependency and lack of validation are serious drawbacks to this model, which is why the GAMBIT is hardly used nowadays. Next to the GAMBIT, the head injury power (HIP) criterion proposed by Newman et al. ${ }^{(15)}$ is an extension of the HIC, calculating the sum of the power (or mechanical energy per unit of time) along each degree of freedom of the head:

$$
\begin{aligned}
H I P & =m a_{x} \int a_{x} d t+m a_{y} \int a_{y} d t+m a_{z} \int a_{z} d t \\
& +I_{x x} \alpha_{x} \int \alpha_{x} d t+I_{y y} \alpha_{y} \int \alpha_{y} d t+I_{z z} \alpha_{z} \int \alpha_{z} d t,
\end{aligned}
$$

with $m$ the mass, $a_{i}$ and translational and rotational acceleration, respectively, along one of the axes and $I_{i i}$ the moments of inertia. Kleiven et al. ${ }^{(16)}$ proposed the Power Index (PI) and more specifically, the PI for predicting ASDH, which adds directional sensitivity to the HIP, by adding scaling coefficients to the various directions and differentiating between positive and negative accelerations:

$$
\begin{aligned}
P I & =\left[C_{1}^{+} \theta\left(a_{x}\right)+C_{1}^{-} \theta\left(-a_{x}\right)\right] m a_{x} \int a_{x} d t+C_{2} m a_{y} \int a_{y} d t \\
& +\left[C_{3}^{+} \theta\left(a_{z}\right)+C_{3}^{-} \theta\left(-a_{z}\right)\right] m a_{z} \int a_{z} d t+C_{4} I_{x x} \alpha_{x} \int \alpha_{x} d t \\
& +\left[C_{5}^{+} \theta\left(\alpha_{y}\right)+C_{5}^{-} \theta\left(-\alpha_{y}\right)\right] I_{y y} \alpha_{y} \int \alpha_{y} d t+C_{6} I_{z z} \alpha_{z} \int \alpha_{z} d t,
\end{aligned}
$$

with $\theta(a)$ the Heaviside's step function defined by:

$$
\theta(a)=\left\{\begin{array}{l}
1, a>0 \\
0, a<0
\end{array}\right.
$$

The scaling factors $C_{i}$ were found by comparing the maximal value of strain in the bridging veins during finite element simulations (see section 5) of the nine relevant acceleration pulses to the head, whereby each separate acceleration pulse corresponded to a maximum HIP value of $17.3 \mathrm{~kW}$. Hence, though still only moderately accurate for combined loadings, the PI already results in an improved prediction of the risk of bridging vein rupture for each of the 
degrees of freedom. His results clearly showed how anterior-posterior (AP) and especially posterior-anterior (PA) rotation by far result in the largest bridging vein strains.

An actual tolerance criterion for bridging vein laceration sets a threshold value at which the veins rupture. The determination of this threshold, particularly for rotation in the PA direction, has been done by a number of research groups, either through cadaveric experiments or by computer simulations combined with tensile tests on the actual bridging veins. The following thresholds have been found in the literature:

- Löwenhielm et al. found a maximum tolerable PA rotational acceleration of $4500 \mathrm{rad} / s^{2}$, through head-on collision experiments with whole cadavers, with a pulse duration between 15 to $44 \mathrm{~ms}^{(17)}$.

- Complementary to the above criterion, for pulse durations shorter than 10ms, Depreitere et al. stated a maximum tolerable PA rotational acceleration of $10000 \mathrm{rad} / \mathrm{s}^{2}$. This was found through cadaver testing ${ }^{(12)}$ where human cadaver heads were impacted by a pendulum.

- Kleiven et al. found a maximum PA rotational acceleration of 34000 $\mathrm{rad} / \mathrm{s}^{2}$ at a $5 \mathrm{~ms}$ pulse duration, by combining the results of their 3D finite element head impact simulations (described in section 5) with the tensile failure tests on bridging veins performed by Lee et al. ${ }^{(18)}$ (described in section 4.4)

- Huang et al. ${ }^{(11)}$ found a PA rotational acceleration limit of 71200 $\mathrm{rad} / \mathrm{s}^{2}$ at a $3.5 \mathrm{~ms}$ pulse duration, by combining the results of their 3D finite element head impact simulations (also described in section 5) with the same tensile failure tests.

Apart from the fact that the tolerance level clearly decreases for longer pulse durations, a second reason for the large differences in the reported values can be found in the difference in testing approach. During cadaveric head impact experiments the real life conditions (impact situation, fresh cadaver material, ...) will be emulated as good as possible. However, biological tissue decay of cadaveric material can never be fully avoided so that the mechanical response will change with increasing postmortem time, making reproducibility limited. Moreover, it is very difficult to apply a purely rotational acceleration through experiments. Likely, a combined loading situation will have taken place. 
On the other hand, finite element simulations do not struggle with tissue decay or non-reproducibility, and are capable of applying pure loads along a single degree of freedom. Finite element models can also predict complex head responses, by taking into account differences of material properties both regional and individual ${ }^{(19)}$. This could potentially lead to the creation of region specific injury tolerance limits ${ }^{(20)}$. However, FE models do rely on accurate definition and validation of the entire model and on correct experimental information regarding rupture values of the bridging vein. This entails correct geometrical and anatomical information together with biofidelic material property assignments. Therefore, the following sections provide a literature review of these topics.

\section{General structure of bridging veins}

\subsection{Anatomy}

Bridging veins (BV) drain the venous blood from the cerebral cortex into the superior sagittal sinus (SSS) and doing so they bridge the subdural space, i.e. the space between the dura mater and the $\operatorname{arachnoid}^{(21)}$ (see fig.1). The cranial end of the bridging veins is fixed to the dura mater whereas the cerebral end is fixed to the movable hemispheres. Unlike the subarachnoidal space where there are arachnoid trabecules, the virtual subdural space provides no additional structural support to the bridging veins. Lateral movement of the brain is minimized by the presence of the falx, i.e. a strong fold of dura mater that descends vertically in the longitudinal fissure between the cerebral hemispheres. However, there is no protection against antero-posterior movement ${ }^{(22)}$. Therefore, an impact to the head causing such an anteroposterior rotation can lead to rupture of the bridging veins mostly. This rupture causes bleeding that dissects the virtual subdural space where the blood is also collected ${ }^{(18)}$.

The direction of inflow from the bridging veins into the SSS shows high variability, and was first described by Oka et al. ${ }^{(24)}$. Five categories can be defined to describe this ${ }^{(25)}$, i.e. (see fig. 2):

- antegrade, i.e. along the flow direction of the SSS,

- perpendicular,

- retrograde, i.e. opposed to the flow direction of the SSS, 


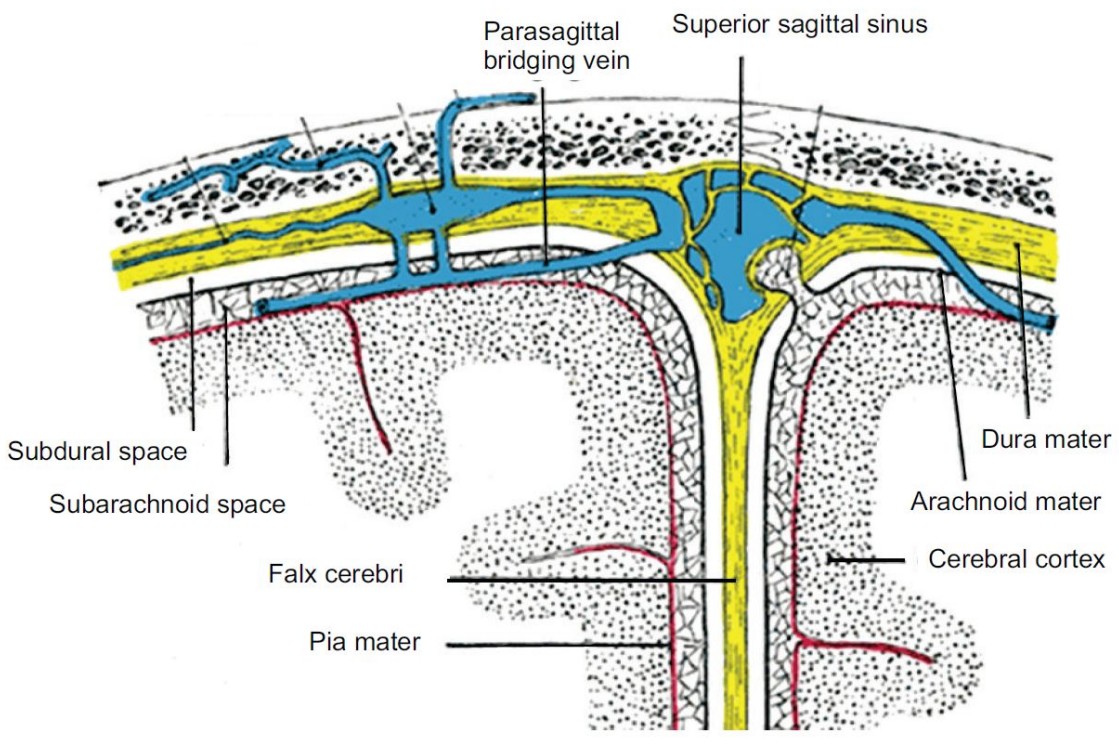

Figure 1: Coronal section through skull, meninges and brain. (adapted from ${ }^{(23)}$ )

- hairpin shaped, i.e. changing direction shortly before entering the sinus,

- lacunae, i.e. enlarged venous spaces.

The entrances of the bridging veins along the SSS are not evenly distributed. Han ${ }^{(26)}$ divided the SSS into four segments from anterior to posterior, where the first two segments have an average length of about $5 \mathrm{~cm}$, whereas the last two measure about $7 \mathrm{~cm}$. Segments 1 and 4 show clusters of incoming BVs, whereas segment 2 and 3 receive only a few. Instead of dividing the SSS into segments, Brockmann ${ }^{(25)}$ used a physical landmark, the coronary suture, to describe his findings of the BVs. For BVs draining into the SSS at the level of the coronary suture, there is a dominant lacunae formation, followed by hairpin-shaped inflow. Posterior to the coronary suture, at the level of segment 3, the inflow direction of the BVs is mainly retrograde with an average angle of $35^{\circ}(26,25)$. For the inflow directions anterior to the coronary suture, the authors state different observations. Han ${ }^{(26)}$ notes that BVs entering the SSS at the level of segment 1 have various inflow directions: roughly 20\% antegrade, $40 \%$ perpendicular and $40 \%$ retrograde. In contrast, Brockmann observed a predominant antegrade inflow direction 
about $2 \mathrm{~cm}$ anterior of the coronary suture ${ }^{(25)}$. Due to the various inflow directions into the SSS a relative motion of the brain with respect to the skull in any direction will result in tensile and shear loading of at least some of the BVs, causing possible lesions ${ }^{(26)}$.

The average outer diameter of a bridging vein is between 0.5 and $5.3 \mathrm{~mm}$ which varies significantly according to the location along the vein. Table 1 gives an overview of the anatomical description of the BVs according to the findings of different authors. The diameter remains more or less constant along its subarachnoid course but increases just before a short and narrow region called the outflow cuff segment. This results in a 'puffy-looking' vein. This phenomenon, described by Vignes et al. ${ }^{(21)}$, is illustrated in figure 3. According to Pang et al. ${ }^{(27)}$, the outflow cuff segment is connected to the SSS through the ampullar bulging, illustrated in figure 4. Although these two findings seem to be describing a different section of the BV-SSS complex, they neither contest or confirm each other's results. Further research is therefore required to obtain clarity. Similar to the outer diameter, a wide range of findings for the length and wall thickness of the BVs have been reported, as shown in table 1.

\subsection{Histology}

Though not always clearly distinguishable in bridging veins ${ }^{(32)}$, the general structure of a venous wall consists of three layers ${ }^{(33)}$ :

- The strong tunica adventitia covers the outside of the vein. This layer is composed of connective tissue that consists of an abundance of collagen and elastin fibres with some elongated mesenchymal cells ${ }^{(22)}$. These fibres protect the vein from overextension.

- The second layer is called the tunica media, consisting of smooth muscle cells and elastin fibres. Concerning the smooth muscles cells there are different findings: Yamashima and Vignes both state that bridging vein walls have smooth muscle cells ${ }^{(22,21)}$, whereas Pang and Kiliç observe that there is an absence of the smooth muscle layer ${ }^{(27,34)}$. Pang used porcine bridging veins whereas Yamashima, Vignes and Kiliç used human specimens. The absence of smooth muscle cells would imply that the regulation of blood flow in the vein and cuff segment happens passively ${ }^{(27)}$. 


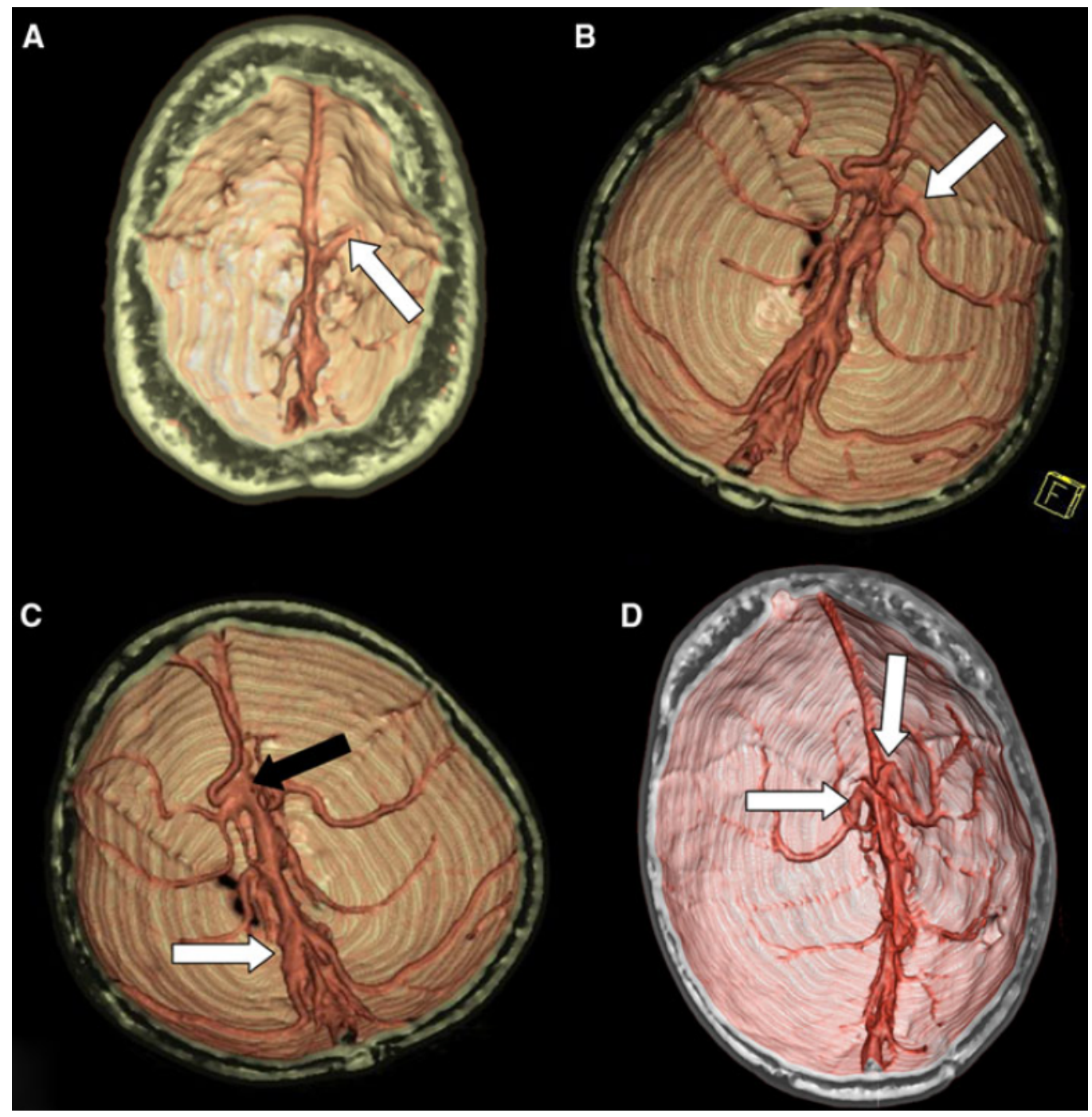

Figure 2: Volume rendering of a venous CTA expressing typical endings: antegrade (a), perpendicular (b), and retrograde (c, white arrow) emptying BV. A lacunae formation is shown on c (black arrow). d shows a hairpin shaped discharging $B V^{(25)}$ 
Table 1: Comparison of SSS-BV anatomical parameters

\begin{tabular}{|c|c|c|c|}
\hline Author & Length (mm) & OD (mm) & $\overline{\mathrm{WT}}(\mathrm{mm})$ \\
\hline Yamashima et al. $(1984)^{(22)}$ & $10-20$ & $1-3$ & $\begin{array}{r}\text { Subdural: } \\
0,01 \pm 0,6 \\
\text { Subarachnoid: } \\
0,05-0,2\end{array}$ \\
\hline Oka et al. $(1985)^{(24)}$ & $0-70$ & $0,5-5,3$ & - \\
\hline Lee et al. $(1989)^{(18)}$ & $6,42 \pm 4$ & $1,4 \pm 0,63$ & $0,05 \pm 0,02$ \\
\hline Sampei et al. $(1996)^{(28)}$ & - & $0,5-4$ & - \\
\hline Ehrlich et al. $(2003)^{(28)}$ & - & $1,4-3,1$ & - \\
\hline Monson et al. $(2005)^{(29)}$ & - & $1,84 \pm 0,35$ & $0,12 \pm 0,02$ \\
\hline \multirow[t]{4}{*}{ Delye et al. $(2006)^{(30)}$} & Male: & Male: & Male: \\
\hline & $22,11 \pm 7,6$ & $2,7 \pm 0,85$ & $0,03 \pm 0,01$ \\
\hline & Female: & Female: & Female: \\
\hline & $17,92 \pm 5,93$ & $2,71 \pm 1,06$ & $0,04 \pm 0,02$ \\
\hline Vignes et al. $(2007)^{(21)}$ & - & $0,5-4$ & - \\
\hline \multirow[t]{3}{*}{ Han et al. (2007) ${ }^{(26)}$} & & Cadavers: & \\
\hline & - & $\begin{array}{r}2,5 \pm 1,1 \\
\text { DSA: }\end{array}$ & - \\
\hline & - & $3,4 \pm 0,8$ & - \\
\hline Monea et al. $(2014)^{(31)}$ & & $3,42 \pm 1,18$ & $0,044 \pm 0,017$ \\
\hline $\mathrm{OD}=$ Outer Diam & Thickness, DS & Digital $\mathrm{Su}$ & on Angiography. \\
\hline
\end{tabular}

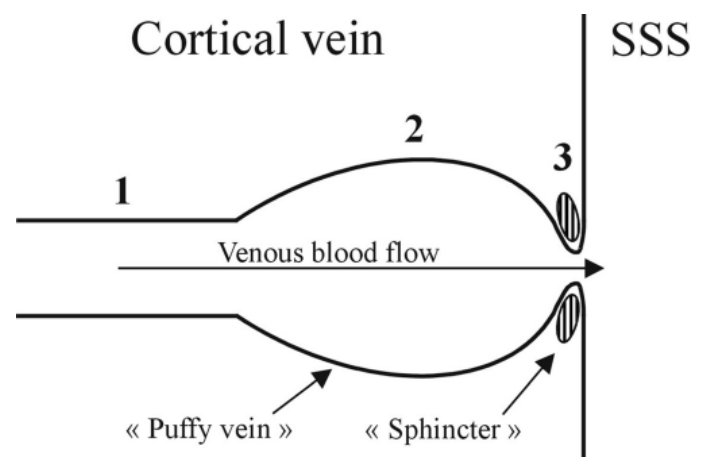

Figure 3: Vignes' observation of the increase of the diameter of the cortical veins just before their opening into the SSS, producing a 'puffy-looking' vein ${ }^{(21)}$. 


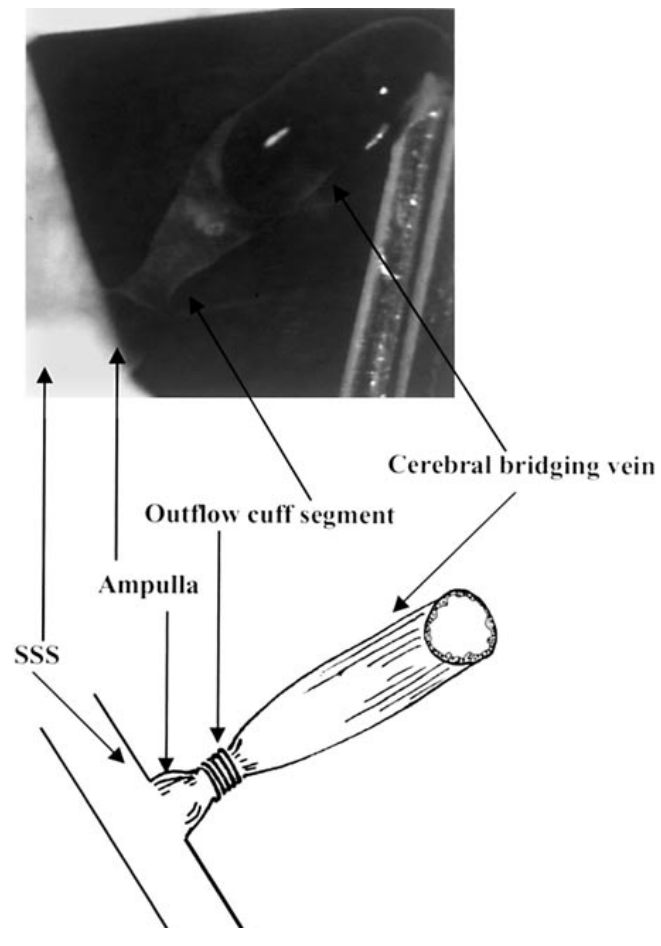

Figure 4: A photograph and schematic illustration of the superior sagittal sinus (SSS), the ampulla, the outflow cuff segment and the cerebral bridging vein according to Pang. ${ }^{(27)}$ 
- Finally, the inner layer or tunica intima is composed of multi-layered smooth endothelium covered by elastin tissue $\mathrm{e}^{(22,35)}$.

The distribution and orientation of the collagen fibres show significant differences depending on the location along the BV. In the subdural portion the collagen fibres show a loose webbing pattern whereas in the subarachnoid portion they have a more dense structure ${ }^{(22,36)}$. Moreover, the collagen fibres are mainly oriented in the longitudinal direction whereas in the outflow cuff segment, the main direction is circumferential ${ }^{(27,32)}$ or helicoidal $^{(21)}$. A circumferential orientation of the collagen fibres in the outflow cuff segment limits its extension to a certain diameter. This limitation gives a dynamic resistance against sudden changes in blood flow ${ }^{(37)}$. In other words, the outflow cuff segment acts as a valve and regulates the flow in the bridging veins according to the Venturi effect ${ }^{(27,21)}$.

Vignes et al. is the only one to observe the presence of abundant smooth muscle cell fibres at the level of the outflow cuff segment ${ }^{(21)}$ and uses the term 'smooth sphincter' for the description of the outflow cuff segment. Hence, they also appreciate its important mechanical role in the control of venous blood flow but have different observations concerning the histological source of it.

\subsection{Characterisation techniques}

\subsubsection{Anatomical characterisation}

Section 3.1 describes the anatomy of the bridging veins, i.e. their macrostructural properties. This paragraph describes experimental techniques used in the literature to obtain this structural overview.

\section{Dissection}

One of the oldest anatomical characterisation techniques is dissection, i.e. the process where the body is disassembled and a certain region of interest is observed with the naked eye. For investigation of the BVs, the region of interest is a section of the brain and/or skull with embedded BVs. Prior to dissection, a pre-treatment can be performed by injection of an enhancing or fixative agent to visualise the veins. A detailed dissection of the brain is described by Oka ${ }^{(24)}$. One may attempt to dissect the venous vasculature (SSS + BVs + cortical cerebral veins) with the escorting meningeal folds from the brain hemispheres, though no precise procedure has been described in the literature. A complete dissection would allow a closer look at the SSS-BV complex separately. 


\section{CT and MRI}

Computed tomography or CT uses the X-ray attenuation properties of the body to produce 2D cross-sectional images, at a resolution up to $82 \mu \mathrm{m}^{(38)}$. Magnetic resonance imaging or MRI uses the physical phenomenon of nuclear magnetic resonance to image nuclei of hydrogen atoms inside the body. This technique can be applied to obtain anatomical images of all parts of the human body that contain hydrogen such as soft tissue, cerebrospinal fluid, etc. For clinical application, 1.5T and 3T systems are used. The resolution of a high-resolution MRI image can go up to 0.5 to $1.0 \mathrm{~mm}$ for a $1.5 \mathrm{~T}$ system and double for a $3 \mathrm{~T}$ system $^{(39)}$. In research applications, MRI studies have been performed up to $9.4 \mathrm{~T}^{(40)}$ on humans and $21.1 \mathrm{~T}$ on animals ${ }^{(41)}$ where the resolution can go up to $100 \mu \mathrm{m}$.

Imaging of the venous system with both CT or MRI requires the injection of a contrast fluid into the veins. Since the contrast fluid will propagate through the body, timing is crucial for obtaining proper venographies. For CT, for example, Brockmann used $100 \mathrm{ml}$ of Imeron(R300 ionized agent at a flow rate of $4 \mathrm{ml} / \mathrm{sec}$ to examine humane $\mathrm{BVs}^{(25)}$. Kiyosue et al. ${ }^{(42)}$ used MRI to investigate the SSS-BV complex with $0.1 \mathrm{mmol} / \mathrm{kg}$ gadoliniumchelate (Omniscan) as contrast agent whereas Spuentrup used Gadofosveset as blood pool contrast agent for the detection of cerebral venous thrombosis in pigs ${ }^{(43)}$.

The pros and cons of CT and MRI are widely discussed in literature. On one hand, MRI is a more sensitive technique compared to CT, in the sense that it provides a better contrast between different types of soft tissue ${ }^{(44)}$. CT scans also imply exposure to ionizing radiation and the use of iodinated contrast material which are both detrimental to the health of the patient ${ }^{(45,46)}$. On the other hand CTs are cheaper, quicker and geometrically more precise than $\mathrm{MRI}^{(47,48)}$.

\section{Digital subtraction angiography}

A fluoroscopic technique used in interventional radiology to visualize blood vessels is digital subtraction angiography (DSA) ${ }^{(49)}$. DSA is considered as the reference imaging technique when it comes to (cerebro)vascular imaging $(50,51,46,52,26)$. The image made of the area of interest without the presence of any contrast material is called the mask, which is digitally subtracted from a contrast enhanced image of the 
same area. As a result, the contrast-enhanced structures are visible, free from background elements ${ }^{(49)}$.

\section{Vascular Corrosion Casting}

Vascular corrosion casting is a technique that is very suitable to describe the morphology and anatomical distribution of blood vessels and is performed on cadavers. After blood removal through flushing with saline, the vascular system of interest can be injected with a plastic polymer (e.g. Mercox resin, PU4ii, ... ${ }^{(53,54)}$ ) which fills the blood space and rapidly polymerizes. Afterwards, the tissue is corroded away with an alkali solution (e.g. $\mathrm{NaOH}, \mathrm{KOH}, \mathrm{HCl}, \ldots{ }^{(26)}$ ) leaving the hardened cast that reflects the shape, size, and distribution of the vessels. The cast is in essence a negative replica of the endothelial surface of that particular vessel. Finally, the cast can be examined with light microscopy, electron microscopy or microCT ${ }^{(53,54)}$, rendering digital 3D reconstructions which allow accurate morphometry. The advantage of this technique above anatomical dissection is the preservation of the in vivo geometry since the vessel does not need to be opened, fixed or dehydrated $^{(55)}$.

\subsubsection{Histological characterisation}

Section 3.2 describes the histology of the bridging veins, i.e. their microstructural properties. This paragraph describes experimental techniques used in the literature to obtain this histological information.

\section{Fixation}

Prior to any kind of histological characterisation, the tissue is fixated to prevent autolysis. There are many kinds of fixation techniques ranging from heat fixation, perfusion, etc. to freezing and chemical fixation. The latter is the most suitable for fixating cadaveric tissue since it preserves the specimens in a state that is closest to the in vivo state. The most commonly used fixative is formalin ${ }^{(21)}$.

\section{Staining}

Samples are embedded in paraffin after fixation and slices of approximately $5 \mu \mathrm{m}$ are made. Next, chemical staining is performed to allow identification of the tissue constituents. H\&E (Haematoxylin and Eosin) is the most commonly used, where haematoxylin stains cell nuclei blue and eosin stains connective tissue, cytoplasm and other 
cytoplasmatic components pink or red. Nierenberger used Masson's trichrome, orcein and orcein-hemalun in their histological study of the bridging veins ${ }^{(32)}$. Masson's trichrome produces red keratin and muscle fibers, blue or green collagen and bone, light red or pink cytoplasm, and dark brown to black cell nuclei ${ }^{(56)}$. In venous tissue, collagen fibers can be identified with Sirius red ${ }^{(57)}$ and elastin with Verhoeff's stain or van Gieson's stain ${ }^{(58)}$. Finally, immunohistochemistry may be used to target specific components such as smooth muscle cells ( $\alpha$-SMA and desmin specific antibodies) or elastin (Elastin antibody).

A non-exhaustive list of staining agents is shown in table 2 .

Table 2: Examples of connective tissue stains and their reactions; MTC = Masson's trichrome; $\mathrm{MSB}=$ Martius; Scarlet and Blue; $\mathrm{PTAH}=$ Phosphotungstic acid-haematoxylin; PAS=Periodic acid-Schiff; HPS=Hematoxylin Phloxine Saffron; H\&E=Haematoxylin and

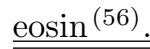

\begin{tabular}{|c|c|c|c|c|}
\hline Stains & Muscle & Collagen & Elastin & Cell nuclei \\
\hline \multicolumn{5}{|c|}{ General staining } \\
\hline H\&E & Deep pink & Deep pink & Pink & Blue \\
\hline HPS & Pink & Yellow & - & Blue \\
\hline \multicolumn{5}{|c|}{ Specific staining } \\
\hline MTC & Red & Blue green & Pale red & Dark red \\
\hline Movat & Red & Yellow & - & Black \\
\hline Sirius red & - & Red & - & - \\
\hline Verhoeff's Stain & Yellow & Red & Black & Black \\
\hline van Gieson & Yellow & Red & Yellow & Black \\
\hline MSB & Red & Blue & Blue & Blue \\
\hline РТАН & Blue & Orange red & Orange brown & blue \\
\hline \multicolumn{5}{|c|}{ Immunostaining } \\
\hline$\alpha$-SMA antibody & + & - & - & - \\
\hline Desmin antibody & + & - & - & - \\
\hline Elastin antibody & - & - & + & - \\
\hline
\end{tabular}

\section{Microscopy}

Stained samples can then be investigated under different kinds of mi- 
croscopes. The most commonly used technique is light microscopy. Light or optical microscopes capture visible light transmitted through or reflected by the sample, offering magnifications up to $100 \mathrm{x}$ and a resolution up to $200 \mathrm{~nm}^{(59)}$. To enhance contrast, polarized light can be used to create differential interference contrast. Collagen (type I) fibres are intensively positively birefringent under polarized light.

Fluorescence microscopy can be used to capture the fluorescent properties of a sample. These properties can be intrinsic to the tissue, e.g. elastin is autofluorescent, or fluorescent staining can be applied, such as the immunostaining explained above.

Further enhancement can be achieved with confocal microscopy. By using point illumination and a spatial pinhole it is possible to focus on a certain depth or layer of tissue without measuring out-of-focus light from other overlaying layers. This significantly increases optical resolution and contrast and enables the reconstruction of a 3D image of the structure. It is used frequently for investigation of vascular tissue (e.g. $\left.{ }^{(60,61,62)}\right)$. The resolution and focal depth increase even further with two-photon laser scanning microscopy ${ }^{(63,64,65)}$. However, due to the limited thickness of these veins, the latter procedures have not been described in the study of cerebral cortical veins.

With electron microscopy, the sample under investigation is illuminated with an electron beam. This results in an extremely high resolution up to $50 \mathrm{pm}$ and magnifications up to $10^{7} \mathrm{x}$, in case the beam is transmitted through the sample (transmission electron microscopy) ${ }^{(66)}$. This has been performed for bridging vein analysis by a.o. Yamashima et al. ${ }^{(22)}$. With scanning electron microscopy, the electron beam does not pass through the sample, but the sample is probed with a focussed electron beam that scans the surface. It can be used to acquire the topology of a vascular corrosion cast. This technique was applied for assessing bridging veins in rats by Auer et al. ${ }^{(67)}$.

\section{Mechanical properties of bridging veins}

The previous section revealed the complex geometrical structure of the bridging vein. Accordingly, the mechanical behaviour of bridging veins is equally complex, and can be described as nonlinear elastic, anisotropic, viscoelastic and prone to rupture ${ }^{(27)}$. The following paragraphs elaborate on 
each of these properties and approaches for their mathematical description as well as methods for mechanical characterization of these properties.

\subsection{Hyperelasticity}

The mechanical behaviour of materials in general can be described by continuum mechanics-based constitutive relations. For biological tissue there have been numerous constitutive relations suggested in literature. These range from the simplest Hookean law to very complex mathematical expressions ${ }^{(68)}$. The description of hyperelastic properties is frequently done by means of a strain energy density function $(\mathrm{SEDF})^{(69)}$. The function defines the potential energy $(\Psi)$ stored in a unit volume of an elastic material as a function of the strain, thus defining a constitutive relation between the three-dimensional second Piola-Kirchhoff stress tensor $(\mathbf{S})$ and the Lagrangian strain tensor $(\mathbf{E})$ :

$$
\mathbf{S}=\frac{\partial \Psi(\mathbf{E})}{\partial \mathbf{E}}
$$

For more details on the kinematics of the strain tensor and its derivatives, see Appendix A.

\subsubsection{Linear elasticity}

The simplest strain energy density function is a purely linear elastic one:

$$
\Psi=\frac{\lambda}{2}\left(\operatorname{tr} \mathbf{E}_{l i n}\right)^{2}+\mu\left(t r \mathbf{E}_{l i n}^{2}\right)
$$

Here, $\lambda$ is Lamé's first parameter and $\mu$ Lamé's second parameter or shear modulus. $E_{l i n}$ is a linearized version of the Lagrangian strain tensor (see Appendix A), which is valid in small strain situations. For linear elasticity, more commonly used material parameters are the Young's modulus $E$ and the Poisson coefficient $\nu$, which relate to the Lamé coefficients as follows:

$$
\begin{array}{ll}
E= & \frac{\mu(3 \lambda+2 \mu)}{\lambda+\mu} \\
\nu & =\frac{\lambda}{2(\lambda+\mu)}
\end{array}
$$

In the one-dimensional case, equations 6,7 and 9 simplify to the well known linear relationship: $\sigma=E \epsilon, \sigma$ being the Cauchy stress and $\epsilon$ the $1 \mathrm{D}$ engineering strain. 
Due to its computational simplicity, linear theory is still widely used for mechanically characterising biological tissue, as a uniaxial tension or compression test suffices to determine $E$ and $\nu$. Monson et al. ${ }^{(70)}$ and Delye et al. ${ }^{(30)}$ conducted mechanical experiments on bridging veins in which a.o. Young's moduli were identified as 6,43 $\pm 3,44 \mathrm{MPa}$ and 30,69 \pm 19,40 MPa, respectively (See also table 3).

\subsubsection{Nonlinear elasticity}

As described in section 3.2, it is shown that collagen and elastin are the major passive mechanical components of the vessel wall ${ }^{(71)}$. The recruitment of elastin fibres is dominant in the low strain region whereas collagen dominates in high strain loading regimes ${ }^{(71,72,73)}$. Due to the gradual recruitment of initially wavy fibres, the vein stiffens as it is being stretched, causing the nonlinearity.

Numerous nonlinear SEDF's with varying complexity are used for arterial tissue, which, when populated with adequate parameters, could equally be used for veins. The two most commonly used nonlinear isotropic SEDF's for vascular tissue are:

Fung: (74)

$$
\Psi=\frac{c}{2}\left[e^{Q}-1\right]
$$

with

$$
Q=A_{1} E_{11}^{2}+A_{2} E_{22}^{2}+2 A_{3} E_{11} E_{22}+A_{4} E_{12}^{2}+2 A_{5} E_{12} E_{11}+2 A_{6} E_{12} E_{22},
$$

where $c$ and $A_{i}$ are material parameters and the $E_{i j}$ the different components of the Lagrangian strain tensor. ${ }^{(75)}$ uses the Fung model for the cerebral veins in their 3D finite element head model (for more details, see section 5), where the parameters are found based on experimental stress-strain curves obtained by ${ }^{(70)}$.

Neo-hookean: ${ }^{(68)}$

$$
\Psi=\frac{\mu}{2}\left(\bar{I}_{1}-3\right)+\frac{\kappa}{2}(J-1)^{2},
$$

where $\mu$ is the shear modulus described earlier, and $\kappa$ is the bulk modulus, which can be derived from previously defined parameters as $\kappa=\lambda+\frac{2 \mu}{3} \cdot \bar{I}_{1}$ and $J$ are invariants of the Cauchy-Green strain tensor as described in the appendix. It is commonly used to model the ground matrix of arterial tissue ${ }^{(76)}$, as well as of venous tissue ${ }^{(77)}$. 


\subsubsection{Anisotropy}

Section 3.2 has also demonstrated that in vascular tissue, the collagen fibres are oriented along a preferred direction, with different orientations for different locations along the vessel tree. Hence, the mechanical properties of the tissue are orientation-dependent ${ }^{(78)}$. One of the most commonly used SEDF's for cardiovascular tissue that captures this anisotropy tissues is the Holzapfel-Gasser-Ogden model ${ }^{(76)}$. This SEDF is written as the sum of an isotropic component, i.e. the matrix material, and an anisotropic component, i.e. two collagen fibre families which are embedded in the matrix material along a certain preferred direction ${ }^{(76,78)}$ :

$$
\Psi\left(I_{1}, I_{4}^{*}, I_{6}^{*}\right)=\frac{c}{2}\left(I_{1}-3\right)+\frac{k_{1}}{2 k_{2}} \sum_{i=4,6}\left(\exp \left[k_{2}\left(I_{i}^{*}-1\right)^{2}\right]-1\right),
$$

where $c>0$ and $k_{1}>0$ are stress-like parameters, $k_{2}>0$ is a dimensionless parameter, and $I_{1}$ is the first invariant of the right Cauchy-Green tensor. $I_{i}^{*}$ is the pseudo invariant related to the fibre direction and can be written as ${ }^{(78)}$ :

$$
I_{i}^{\star}=\kappa I_{1}+(1-3 \kappa) I_{i}, \quad I_{i}=\lambda_{\theta}^{2} \cos ^{2} \alpha+\lambda_{z}^{2} \sin ^{2} \alpha, \quad i=4,6,
$$

where $\alpha$ is the angle between the (mean) fiber direction and the circumferential direction of the vessel, and $\kappa \in[0,1 / 3]$ is a parameter related to the dispersion of the fibers.

Though this model has mainly been used for modelling arterial tissue ${ }^{(35,79)}$, it can equally be used for modeling the venous wall. It has, for example, been used to model mice vena cava tissue by McGilvray et al. ${ }^{(80)}$ and by Sokolis to model porcine jugular veins ${ }^{(77)}$. No parameters for cerebral bridging veins have been found yet.

Rezakhaniha et al. ${ }^{(81)}$ proposed a similar model specifically for veins, which they applied on the facial veins of New Zealand rabbits. The function is an extension of the Zulliger model ${ }^{(82)}$ and is composed of an isotropic part for the elastin matrix and an anisotropic part for the collagen contribution, according to the Zulliger model, as well as an additional anisotropic part for the anisotropic elastin contribution, which is supposed to contribute specifically in the lower strain region.

Another approach to modeling the anisotropic behaviour of a venous wall is through multiscale modelling, developed for bridging veins by Nierenberger 
et al. ${ }^{(83)}$. Here, the mechanical behaviour of the most important tissue constituents are modelled, starting from the collagen fibril scale, which are bundles of microfibrils. These fibrils constitute the wavy collagen fibres at a higher scale, which in turn constitute the venous wall together with the matrix material at the macroscopic scale. The macroscopic stiffness at the venous wall scale is computed in an incremental process, where the strains at each subscale are computed through homogenization techniques and transitions between the different scales are accounted for through exchange parameters. This approach eliminates non physical parameters in the model. In the case of Nierenberger, a Young's modulus and Poisson coefficient are assumed for the isotropic matrix and for the collagen microfibrils.

\subsection{Viscoelasticity}

Many biological soft tissues have visco-elastic properties ${ }^{(84)}$, which implies a strain rate dependency of the mechanical properties mentioned above. Löwenhielm was one of the first to test this theory ${ }^{(17)}$ for bridging veins. He found that the ultimate strain was reduced from about $70 \%$ to $20 \%$ with increasing strain rate from $1 s^{-1}$ to $1000 s^{-1}$. Later on Gennarelli and Thibault confirmed this statement by their experiments where they induced ASDH in rhesus monkeys ${ }^{(10)}$. However, a later study conducted by Löwenhielm ${ }^{(17)}$ contradicted his first findings, stating the strain rate insensitivity of bovine bridging veins. The strain rate dependency hypothesis was rejected many times more in various later studies ${ }^{(18,85,70,30)}$. They all used dissected bridging veins from human cadavers and found no strain rate effect. Identical conclusions were made in a research conducted on the bridging veins of ferrets $^{(86)}$. Apart from the disputed results of Löwenhielm, the aforementioned studies were conducted only at relatively low strain rates ranging from 0.1 $s^{-1}$ to $3.8 s^{-1}$. Baeck and Monea ${ }^{(87,31)}$ repeated the tests for both low and high strain rates $\left(10.81 s^{-1}\right.$ to $\left.198.6 s^{-1}\right)$ and confirmed the strain rate inde-

pendency. Hence, it is likely that no viscoelastic effects need to be captured in the material model.

\subsection{Residual strains}

For a correct description of the stress-strain behaviour, a stress-free situation has to be taken as reference state. In vascular tissue residual strains are present in both longitudinal and circumferential direction ${ }^{(88,74,89)}$. The stress free situation can be obtained by excising a piece of the vascular tube, to remove the longitudinal residual strain, and cutting open the vessel ring, 
to remove the circumferential residual strain. In the longitudinal direction the residual strain can be quantified by comparing the in vivo length with the length after excision. The residual strain in circumferential direction is quantified by the opening angle $\theta$ as illustrated in fig 5 .

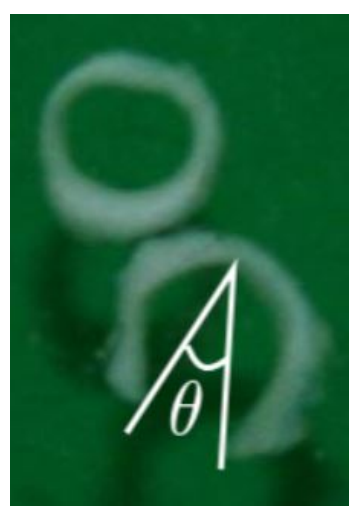

Figure 5: opening angle $\theta$, quantifying the residual strain in circumferential direction ${ }^{(79)}$

Pang ${ }^{(27)}$ conducted opening angle measurements on porcine bridging veins and found the opening angle of the outflow cuff segment and the bridging vein itself to be $115 \pm 4^{\circ}$ and $120 \pm 4^{\circ}$ (means $\pm \mathrm{SE}$ ) without statistical difference between the two regions. To the authors' knowledge, no data is available on the longitudinal residual strains of bridging veins.

\subsection{Failure characteristics}

Excessive load, be it due to head impact or surgical intervention, will cause bridging veins to rupture. Failure properties are commonly expressed with parameters such as ultimate stress, ultimate strain, yield stress and yield strain $\left(\sigma_{U}, \epsilon_{U}, \sigma_{Y}, \epsilon_{Y}\right)$, as shown in fig. 6. The yield stress and strain describe the transition from elastic to plastic deformation for a linear elastic material as described in 4.1.1, i.e., where the material starts to soften. The ultimate stress and strain indicate the actual failure limit. A few studies have reported these parameters for bridging veins, as shown in table 3. Monea et al. ${ }^{(31)}$ also found that the mechanical failure properties were gender-dependent, with female specimens having higher values than the male specimens.

For both Young's modulus and failure characteristics there is a significant difference between Delye's and Monea's results on the one hand and Monson and Lee on the other hand. The reason for these differences might be 
attributed to the dissection method used by Delye and Monea. Contrary to the others, they included a piece of the SSS when collecting the BV samples for testing. Consequently, the SSS-BV outflow cuff segment (OCS) site is included in their studies whereas for the other studies it was not. Referring to the paragraph of histology in this paper, and to the study of Pang, Yamashima and Vignes ${ }^{(27,22,21)}$, the OCS has a distinct morphology and is most probably stiffer compared to the more upstream part of the BV. Though in linear theory a series of springs should result in a less stiff compound, this is not the case in nonlinear theory, as the stiffer region will cause the less stiff region to reach its nonlinear and failure region faster, i.e. at lower strains. This could explain why Delye and Monea report a higher stiffness value, similar ultimate stress, but lower ultimate strain.

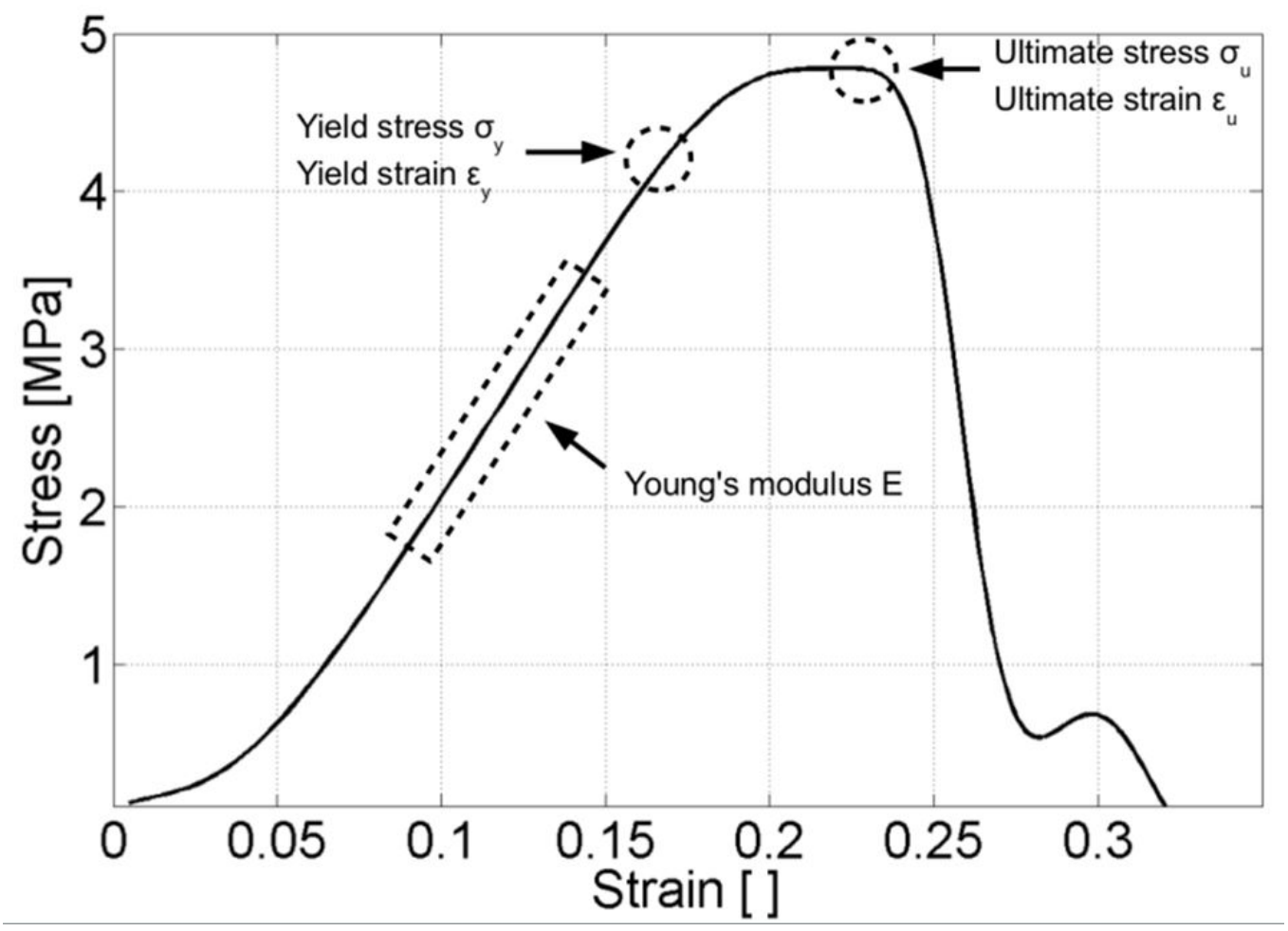

Figure 6: Typical Stress-Strain curves: ultimate stress, ultimate strain, yield stress and yield strain $\left(\sigma_{U}, \epsilon_{U}, \sigma_{Y}, \epsilon_{Y}\right)^{(30)}$

Though useful due to its simplicity, the description of material softening through yield stress and strain is not the most physiologically realistic one. 
Table 3: Comparison of SSS-BV mechanical parameters

\begin{tabular}{crrrrr}
\hline \hline Author & $\sigma_{U}(\mathrm{MPa})$ & $\epsilon_{U}(\%)$ & $\sigma_{Y}(\mathrm{MPa})$ & $\epsilon_{Y}(\%)$ & $\mathrm{E}(\mathrm{MPa})$ \\
\hline $\begin{array}{c}\text { Lee et al. } \\
(1989)^{(18)}\end{array}$ & $3,33 \pm 1,52$ & $53 \pm 19,5$ & - & - & - \\
$\begin{array}{c}\text { Monson et al. } \\
(2005)^{(29)}\end{array}$ & $1,32 \pm 0,62$ & $50 \pm 19$ & $1,15 \pm 0,47$ & $29 \pm 9$ & $6.43 \pm 3,44$ \\
$\begin{array}{c}\text { Delye et al. } \\
(2006)^{(30)}\end{array}$ & $4,99 \pm 2,55$ & $25 \pm 8$ & $4,13 \pm 2,14$ & $18 \pm 7$ & $30.69 \pm 19,40$ \\
$\begin{array}{c}\text { Monea et al. } \\
(2014)^{(31)}\end{array}$ & $4,19 \pm 2,37$ & $29,82 \pm 13,26$ & $1,73 \pm 1,37$ & $12,85 \pm 13,35$ & $25,72 \pm 15,86$ \\
\hline
\end{tabular}

Specifically for cardiovascular tissue, more gradual damage models have been integrated with the nonlinear anisotropic models described earlier ${ }^{(90,91,92)}$. These are yet to be fitted for venous tissue and bridging veins specifically.

\subsection{Testing methods}

So far, bridging veins have mostly been tested in uniaxial tensile set-ups where the specimen is loaded until failure ${ }^{(17,18,86,30,31)}$. This type of set-ups are equipped with load cells measuring the tensile force from which the stress values can then be calculated. Ideally, the test procedure is filmed with a high speed camera. If the samples are marked with dyed reference points, strain levels can be calculated ${ }^{(30,31)}$ more reliably than when merely monitoring actuator displacement.

Obviously, uniaxial testing methods can only lead to one-dimensional properties, unless tests are repeated with samples of different orientations. Even in the latter case, anisotropic material properties can only be estimated properly if histological information on fibre orientation is known ${ }^{(93)}$. Better would be to perform tests that load the tissue in (at least) two dimensions. Planar biaxial tests are performed regularly on cardiovascular tissue samples ${ }^{(94,95,96,97)}$ and could therefore be equally suitable for bridging vein samples. The challenge in these set-ups is the mounting of the sample given the small dimensions, which is usually done by piercing needles through the sample on each side. This method limits the range of strains up to which the sample can be stretched without rupture at the puncture holes. For tubular segments, an extension-inflation test also provides biaxial information, and even loads the tissue in a more physiological manner than a planar biaxial 
set-up, as it allows to pull the sample up to physiological stretch conditions and then inflates it up to a chosen pressure. This type of experiments is commonly performed on arterial tissue ${ }^{(98,79,95,99,100)}$.

Although biaxial testing methods can provide shear properties of the specimen it is challenging if not impossible to test until failure. To allow proper simulation of head impact, it is important that information is acquired on the behaviour of the bridging veins under loading conditions experienced during this head impact. Shear testing methods that allow testing until failure would therefore be valuable. The rail shear test is commonly used for testing composite material to determine shear stresses ${ }^{(101,102)}$. A downscaled version of this test could be used for bridging veins. Another shear testing method in which the vein could remain in its tubular shape is the tube torsion test. This test has already been suggested for arterial segments ${ }^{(95)}$ and might be down-scaled for bridging veins.

\section{Biomechanical modelling of the human head}

As stated in section 2 the definition of accurate tolerance criteria would benefit greatly from finite element (FE) head models. FE simulation is a tool that can provide quantitative data in the investigation of injury mechanics or evaluation of effectiveness of safety equipment such as helmets or air bags. FE head models can predict head responses and injuries for a given impact situation. Given the prevalence of bridging vein rupture following impact, it is essential to have a proper representation of these bridging veins ${ }^{(103,87)}$. Obviously, depending on the geometry and material definition of all the constituents, different models will give different responses. For the geometric design of the FE head models, different sources can be used to retrieve the anthropometric information of the head. These sources can be either anatomy atlases, databases or medical images (MRI or CT). Once the geometry is defined, the model is subdivided into discrete elements and boundary conditions and material properties are applied. The first FE head model was developed by Ward and Thompson ${ }^{(104)}$ and several FE head mod-

els have followed since ${ }^{(103,105,106)}$. This section provides a short overview of those state-of-the-art head models that have included bridging veins.

\section{UDS FE head model}

The Université de Strasbourg (UDS) has developed several FE head models. Some of them, e.g. the infant FE head model, have BVs 
represented as the width of the CSF (length between a node on the inner surface of the skull and a node on the outer surface layer of the brain $)^{(107,108,109)}$. Since the BVs are not mechanically represented they do not contribute to the stiffness of the FE model, but their strain can be computed as the ratio of deformation to their original length.

\section{Shugar model adopted by Huang}

Huang et al. ${ }^{(11)}$ modified the head model of Shugar ${ }^{(110)}$ to study ASDH mechanics upon head impact. The model included a coarsely meshed three-layered skull, the falx, tentorium, foramen magnum and brain, but no CSF. The interface between the skull and the brain was tied. As in the UDS model, an indirect approach was used to account for the BVs, in which the distance between a node on the interior of the skull and a node in the brain, located close to the vertex, was used as a measure of bridging vein deformation.

\section{SIMon}

Takhounts et al. created the simulated injury monitor (SIMon), a FE head model with cerebrum, cerebellum, falx, tentorium, combined piaarachnoid complex (PAC) with cerebro-spinal fluid (CSF), ventricles, brainstem, and bridging veins ${ }^{(111)}$. Here the BVs are modelled as cable discrete beams with a Young's modulus of $0.275 \mathrm{MPa}$, i.e. lower than the values reported in table 3 .

\section{KTH FE head model}

The head model created by Kleiven ${ }^{(7,16,112,75)}$ is shown in figure 7 and includes the scalp, skull, meninges, CSF, cerebrum, cerebellum, brainstem, transverse and superior sagittal sinus, a simplified neck with spinal cord and 11 pairs of bridging veins. The bridging veins were modelled according to the anatomical descriptions of Oka et al. ${ }^{(24)}$, and were included by connecting a node on the cortical surface of the brain to a node on the SSS. These connections are represented as linear spring elements with a stiffness of $1,9 N$ per unit strain, with different lengths depending on the location. This stiffness value was derived from the load-stretch curve presented by Lee and Haut ${ }^{(18)}$ for a representative BV sample. To relate this value to the Young's moduli presented in table 3 one must divide by the cross-sectional area of the element. In the same study, Lee and Haut reported cross-sections ranging from $0.022 \mathrm{~mm}^{2}$ to $0.9 \mathrm{~mm}^{2}$, resulting in a Young's modulus between 2.1 and 
$86 \mathrm{MPa}$. This corresponds to the order of magnitude of the values mentioned in table 3 .

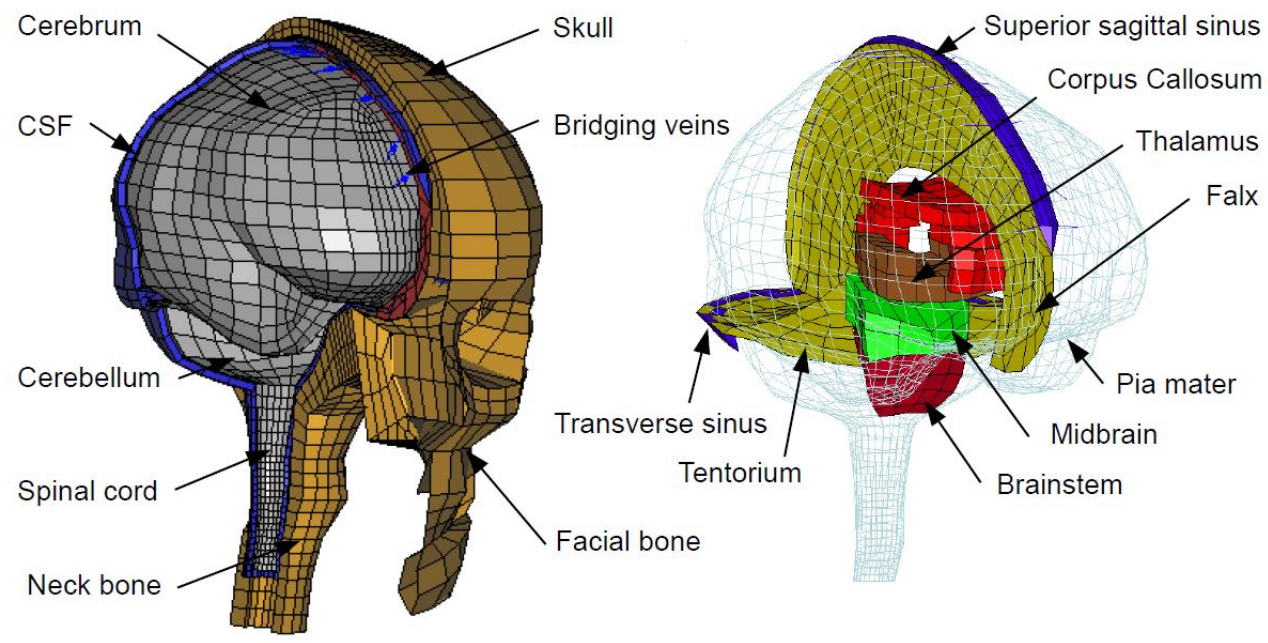

Figure 7: Kungliga Tekniska Högskolan (KTH) finite element head model ${ }^{(75)}$, showing a.o. the bridging vein spring elements.

\section{UCDBTM}

The head model created by Horgan et al. $\left(\mathrm{UCDBTM}^{(6)}\right.$ is shown in figure 8. The anatomical components are: skull, CSF, cerebrum, cerebellum, brainstem, scalp, falx, tentorium, dura and pia mater. and 11 pairs of bridging veins ${ }^{(113)}$. The bridging veins were constructed in a similar way as in the KTH model. A schematic view of the BVs and their orientations is shown in figure 9. Other researchers have also used this model or a modified version for their head impact research ${ }^{(114,115)}$.

\section{WSUBIM}

Several versions of the Wayne State University Brain Injury Model (WSUBIM) have been developed over the past decades. The version presented in ${ }^{(116)}$, and updated and used a.o. in ${ }^{(117,118)}$ contains a.o. the scalp, skull, dura, falx cerebri, tentorium, pia, cerebrospinal fluid (CSF), venous sinuses, ventricles, cerebrum (gray matter and white matter), cerebellum, brain stem, and bridging veins. Ten pairs of bridging veins were included with length of 8 to $17 \mathrm{~mm}$ and orientation of 


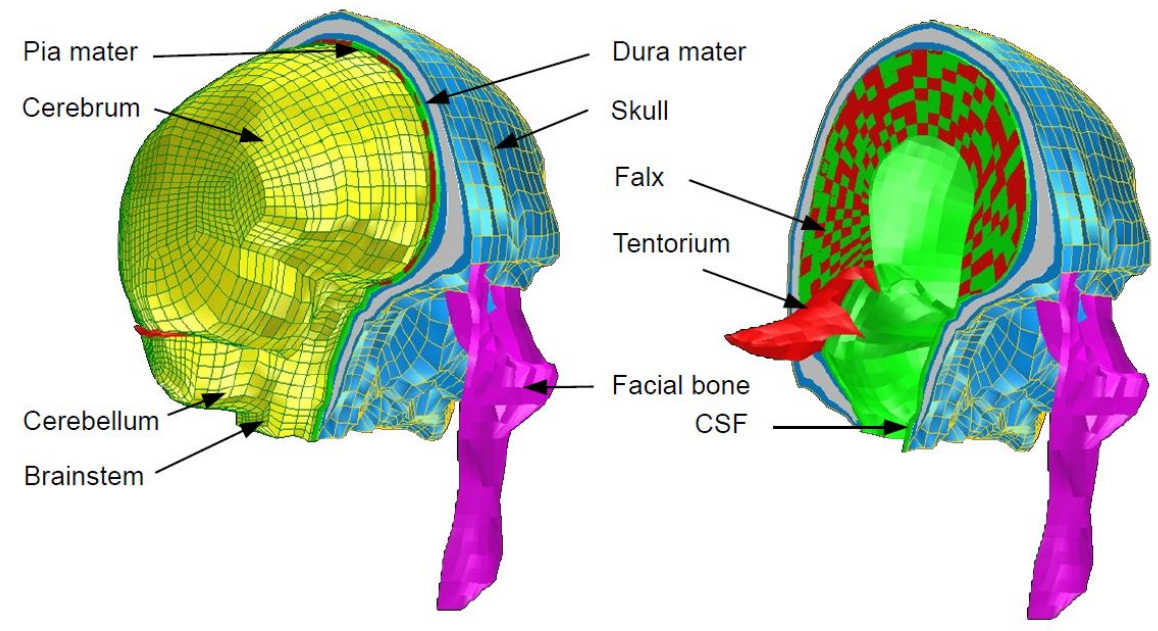

Figure 8: University College Dublin brain trauma model (UCDBTM) ${ }^{(6)}$

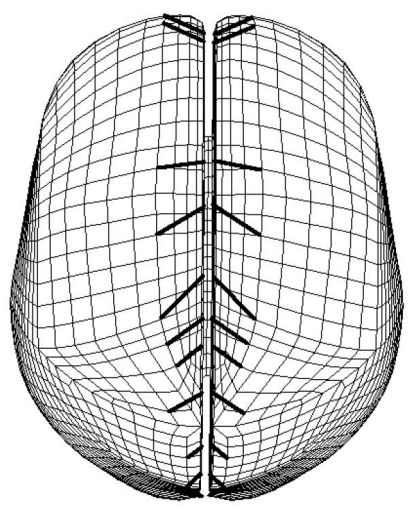

Figure 9: Bridging vein in the $\operatorname{UCBTM}^{(113)}$ 
160 to 1100, again based on the findings of Oka et al. ${ }^{(24)}$, as shown in figure 10. The bridging veins in this model are linear elastic spring elements with a spring constant of $0.219 \mathrm{~N} / \mathrm{mm}$. This spring constant is per definition equal to $\frac{E A}{l}$, with $E$ being the Young's modulus, $A$ the cross-sectional area and $l$ the length. When taking into account the lengths of the BV's and the range of cross-sectional area's reported by Lee and Haut, this corresponds to a Young's modulus between 2 and $169 \mathrm{MPa}$.

Recently, Mao et al. developed an improved FE head model at Wayne State University, based on CT and MR images of an average American adult male, including hexahedral meshes of the brain (cerebrum, cerebellum, brainstem, corpus callosum, ventricles, and thalamus), as well as the bridging veins, cerebrospinal fluid, skull, facial bones, flesh, skin, and membranesincluding falx, tentorium, pia, arachnoid, and dura. It was validated against 35 experimental head impact loading cases. As in the model of Kleiven and Hardy ${ }^{(7)}$, 11 pairs of bridging veins were modelled, with lengths ranging from 6.63 to $17.91 \mathrm{~mm}$, an outer diameter of $2.76 \mathrm{~mm}$ and a wall thickness of $0.03 \mathrm{~mm}$. For the material properties, the values found by Delye et al. ${ }^{(30)}$ (see table 3 ) were used.

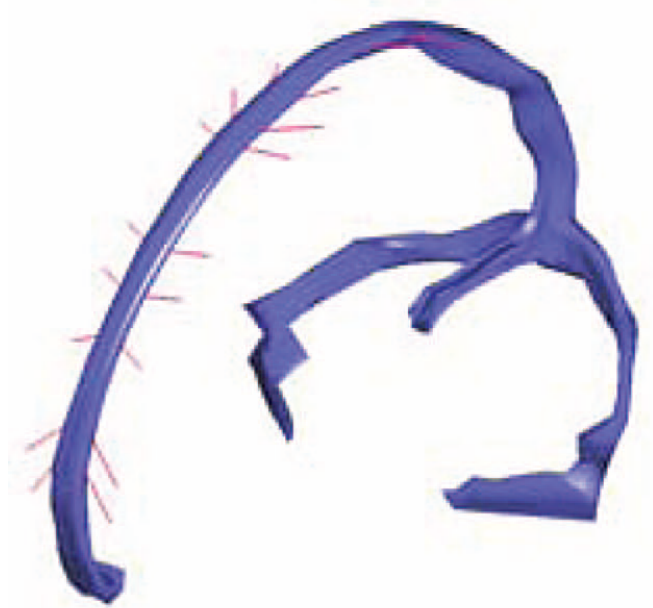

Figure 10: Detail of the bridging veins and the SSS in the WSUBIM, adapted from ${ }^{(118)}$

\section{Global/local head model}

Zoghi-Moghadam et al. have proposed a global/local head modeling 
approach to study bridging vein rupture in more detail ${ }^{(119)}$. A global solid model (GSM) was constructed representing the skull, brain and meningeal layers. This model is based on the FE head model proposed by Horgan and Gilchrist ${ }^{(6)}$ and calculates the stresses and strains in the head. Secondly a global fluid model (GFM) of the CSF and subarachnoid space, in which the skull and brain are considered as rigid boundaries, was designed to calculate the change in fluid pressure of the CSF. The outputs of these two models were then used in a third smaller but more detailed local solid model (LSM) out of which the strains of the BVs were determined. The LSM represents a $6 \mathrm{~mm} \times 6 \mathrm{~mm}$ area of the skull that is cut through the head. The model includes the skull, dura mater, arachnoid mater, subarachnoid space, randomly oriented trabeculae, blood vessels (one artery and one bridging vein), pia mater and the brain, as shown in figure 11. A Young's modulus of 4.6 MPa was used for the bridging vein, which is consistent with the values found by Monson et al. ${ }^{(29)}$. The boundary conditions of the LSM follow directly from the results calculated in the GSM and the GFM. By subjecting the two global models to two sets of impact loadings with HIC values of 740 and 1044 respectively they found the second impact to be strong enough to predict BV rupture according to the experimental ultimate strain values of Lee et al. and Monson et al. (see table 3).

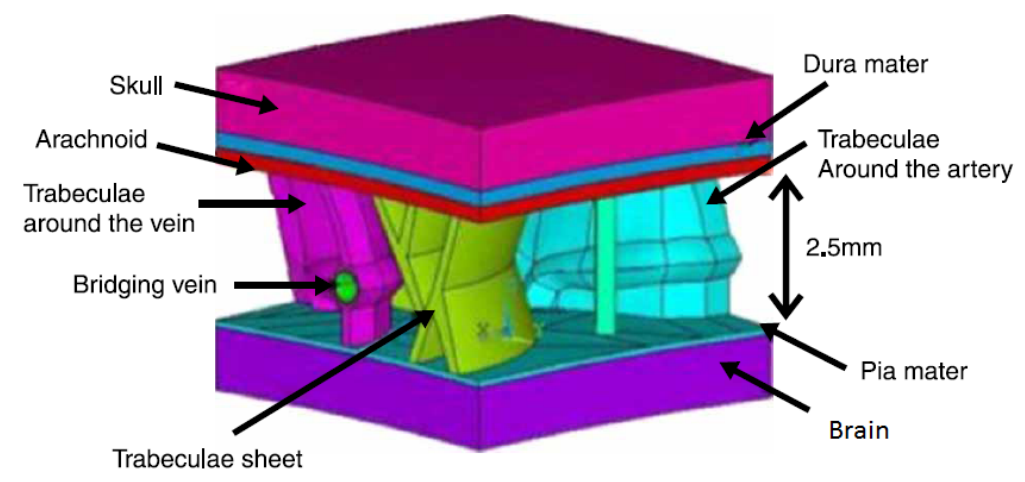

Figure 11: Schematic of the LSM model of Zoghi-Moghadam, containing a bridging vein, adapted from $^{(119)}$. 


\section{Conclusion \& future work}

The mechanopathology of acute subdural haematomas is undoubtedly important with its mortality rate of $30 \%$ to $90 \%$. Knowing that one third of these lesions are caused by a rupture of the bridging veins makes the thorough investigation of these veins only a logical step in the development of a tolerance criterion for ASDH and, ultimately, the development of safer head protective devices. Section 2 showed how different tolerance criteria have been suggested, either based on experimental observations, or based on FE modeling. Experimental observations suffer from a lack of repeatability, whereas FE simulations still lack proper representation of the bridging veins. Hence, a correct anatomical and mechanical characterization and subsequent implementation in a finite element head model is indispensible to actually reach a consensus on a tolerance criterion for bridging vein rupture.

Several groups have performed anatomical and histological investigations on bridging veins, using various techniques such as anatomical dissection, histology, imaging through CT and MRI and vascular corrosion casting, as explained in section 3.3. Anatomically, the SSS-BV complex can roughly be divided into three parts: the bridging vein, that constitutes the last part of the cortical venous system before entering the SSS, the outflow cuff segment which is a narrowing of the BV such that it acts both as shock absorber and as ejection cone, and thirdly the ampulla, which is the site at which the BVs connect to the SSS. All the BVs drain into the SSS from different angles, depending on their location along the SSS. Also the length, thickness and diameter of the BVs vary significantly depending on their location. Due to the various directions the BVs have before entering the SSS a relative motion of the brain with respect to the skull in any direction will result in tensile and shear loading of the BVs, causing possible lesions ${ }^{(26)}$. From a histological point of view the most remarkable feature is a more or less longitudinal orientation of the collagen fibres in the BVs and a circumferential or helical orientation in the outflow cuff segment. Consequently the BV can expand radially just before the outflow cuff segment and has a restricted diameter at the level of the outflow cuff segment itself. This makes this section function as both a shock absorber and as ejection cone. No consensus has been reached on whether or not BVs contain smooth muscle cells.

Concerning the mechanical properties of BVs, so far, only uniaxial tensile tests have been reported. These have aided in the determination of the typical linear elastic and failure parameters, namely Young's modulus, ulti- 
mate stress, ultimate strain, yield stress and yield strain (E, $\left.\sigma_{U}, \epsilon_{U}, \sigma_{Y}, \epsilon_{Y}\right)$. However, due to the difference in testing methods, the results vary strongly between research groups. As for the visco-elastic properties different authors agree on the strain rate insensitivity of the mechanical properties of the BVs. The linear elastic properties described are in fact only valid in small strain situations, which is clearly not the case when BV rupture occurs. Also obvious from the histological description is the presence of collagen, which renders the bridging veins nonlinear, due to the gradual recruitment of the fibers when stretched, and anisotropic, due to the preferential orientation of the fibers. Though not yet fitted for BVs, suitable nonlinear anisotropic material models do exist. These could be populated with realistic parameters if biaxial experiments were performed, or a combination of uniaxial and shear tests. Another approach to capture the collagen-related features is through multiscale modeling, which requires a thorough understanding of the properties of the collagen microfibrils and of the exact morphological composition.

Section 5 presented an overview of state-of-the-art FE head models that do include some form of BV's. The most basic representation is a purely geometrical one, where the BVs do not contribute mechanically but their elongation is assumed by considering the change in distance between their insertion points for a certain loading situation. Slightly more correct implementations do assign a linear stiffness value to the BV elements, but disregard variations in length and cross-sectional area, which results in quite severe over- and underestimation of the actual stiffness value depending on the specific size and location of the BV. Even more correct implementations do take the correct morphological characteristics of the BVs into account, but still assign linear stiffness properties to the material.

Further research should be directed towards the full morphological and histological characterisation of the SSS-BV complex, to rule out current discrepancies between different studies regarding geometry and constitution. Secondly, mechanical tests should be performed that enable the characterization of the anisotropic and nonlinear mechanical properties of the BV and allow a consensus to be reached on the failure characteristics. Subsequently, existing finite element models can be improved with the correct material properties and geometrical information, such that the behaviour of the SSSBV complex during head impacts can be simulated in a more biofidelic manner. This will contribute to a better understanding of the mechanopathology of ASDH and the formulation of proper tolerance criteria for the development of protective head devices. 


\section{Acknowledgements}

This work was supported by a $\mathrm{PhD}$ grant from the Institute for the Promotion of Innovation through Science and Technology in Flanders (I.W.T.Vlaanderen), by an FWO postdoctoral fellowship, by an FWO-Levenslijn project (G.0C67.13) and by a research grant of the KU Leuven (3E120264).

\section{References}

[1] C. Van Lierde, Biomechanics of head injury damage criteria for skull and brain lesions, Phd thesis, Katholieke Universiteit Leuven (2005).

[2] B. Depreitere, C. Van Lierde, S. Maene, C. Plets, J. Vander Sloten, R. Van Audekercke, G. Van der Perre, J. Goffin, Bicycle-related head injury: a study of 86 cases., Accident; analysis and prevention 36 (4) (2004) 561-7.

[3] B. Depreitere, C. Van Lierde, J. Vander Sloten, R. Van Audekercke, G. Van der Perre, C. Plets, J. Goffin, Mechanics of acute subdural hematomas resulting from bridging vein rupture., Journal of neurosurgery 104 (6) (2006) 950-6.

[4] A. G. Monea, G. Van der Perre, K. Baeck, H. Delye, P. Verschueren, E. Forausebergher, C. Van Lierde, I. Verpoest, J. Vander Sloten, J. Goffin, B. Depreitere, The relation between mechanical impact parameters and most frequent bicycle related head injuries., Journal of the mechanical behavior of biomedical materials (2013) 1-13.

[5] H. Maxeiner, Detection of ruptured cerebral bridging veins at autopsy., Forensic science international 89 (1-2) (1997) 103-10.

[6] T. J. Horgan, M. D. Gilchrist, The creation of three-dimensional finite element models for simulating head impact biomechanics, International Journal of Crashworthiness 8 (4) (2003) 353-366.

[7] S. Kleiven, Finite element modeling of the human head, Phd thesis, Royal University of Technology (2002).

[8] J. Versace, A review of the severity index, in: 15th Stapp Car Crash Conference, 1971. 
[9] T. Gennarelli, L. Thibault, Ommaya AK, Pathophysiologic responses to rotational and translational accelerations of the head, Stapp car crash journal 16 (1972) 296-308.

[10] T. Gennarelli, L. Thibault, Biomechanics of acute subdural hematoma, Journal of trauma 22 (1982) 680-686.

[11] H.-M. M. Huang, Three-Dimensional Finite Element Analysis of Subdural Hematoma, Journal of Trauma-Injury Infection \& Critical Care (1999) 538-544.

[12] B. Depreitere, A rational approach to pedal cyclist head protection, Ph.D. thesis, Katholieke Universiteit Leuven (2004).

[13] P. Verschueren, Biomechanical analysis of head injuries related to bicycle accidents and a new bicycle helmet concept, Phd thesis, Katholieke Universiteit Leuven (2009).

[14] J. Newman, a generalized acceleration model for brain injury threshold (GAMBIT), in: Proc. of the International IRCOBI Conf. on the Biomechanics of Impacts, 1986.

[15] J. A. Newman, N. Shewchenko, E. Welbourne, A proposed new biomechanical head injury assessment function - the maximum power index., Stapp car crash journal 44 (2000) 215-47.

[16] S. Kleiven, Influence of impact direction on the human head in prediction of subdural hematoma., Journal of neurotrauma 20 (4) (2003) 365-79.

[17] P. Löwenhielm, Dynamic properties of the parasagittal bridging veins., Zeitschrift für Rechtsmedizin. Journal of legal medicine 74 (1) (1974) $55-62$.

[18] M. C. Lee, R. C. Haut, Insensitivity of tensile failure properties of human bridging veins to strain rate: implications in biomechanics of subdural hematoma., Journal of biomechanics 22 (6-7) (1989) 537-42.

[19] A. I. King, K. H. Yang, L. Zhang, W. Hardy, D. C. Viano, Is head injury caused by linear or angular acceleration?, in: IRCOBI Conference, no. September, Lisbon (Portugal), 2003, pp. 1-12. 
[20] A. Post, T. B. Hoshizaki, Mechanisms of brain impact injuries and their prediction: A review, Trauma 14 (4) (2012) 327-349.

[21] J.-R. Vignes, A. Dagain, J. Guérin, D. Liguoro, A hypothesis of cerebral venous system regulation based on a study of the junction between the cortical bridging veins and the superior sagittal sinus. Laboratory investigation., Journal of neurosurgery 107 (6) (2007) 1205-10.

[22] T. Yamashima, R. L. Friede, Why do bridging veins rupture into the virtual subdural space?, Journal of neurology, neurosurgery, and psychiatry 47 (2) (1984) 121-7.

[23] H. Gray, Gray's Anatomy: The Anatomical Basis of Medicine and Surgery, Elsevier, Churchill Livingstone, 2008.

[24] K. Oka, A. J. Rhoton, M. Barry, R. Rodriguez, Microsurgical anatomy of the superficial veins of the cerebrum, Journal of Neurosurgery 17 (1985) 711-748.

[25] C. Brockmann, S. Kunze, J. Scharf, Computed tomographic angiography of the superior sagittal sinus and bridging veins., Surgical and radiologic anatomy : SRA 33 (2) (2011) 129-34.

[26] H. Han, W. Tao, M. Zhang, The dural entrance of cerebral bridging veins into the superior sagittal sinus: an anatomical comparison between cadavers and digital subtraction angiography., Neuroradiology 49 (2) (2007) 169-75.

[27] Q. Pang, X. Lu, H. Gregersen, G. von Oettingen, J. Astrup, Biomechanical properties of porcine cerebral bridging veins with reference to the zero-stress state., Journal of vascular research 38 (1) (2001) 83-90.

[28] T. Sampei, N. Yasui, T. Okudera, H. Fukasawa, Anatomic study of anterior frontal cortical bridging veins with special reference to the frontopolar vein., Neurosurgery 38 (5) (1996) 971-5.

[29] K. L. Monson, W. Goldsmith, N. M. Barbaro, G. T. Manley, Significance of source and size in the mechanical response of human cerebral blood vessels., J Biomech 38 (4) (2005) 737-744. 
[30] H. Delye, J. Goffin, P. Verschueren, J. Vander Sloten, G. Van der Perre, H. Alaerts, I. Verpoest, D. Berckmans, Biomechanical properties of the superior sagittal sinus-bridging vein complex., Stapp car crash journal 50 (November) (2006) 625-36.

[31] A. G. Monea, K. Baeck, E. Verbeken, I. Verpoest, J. V. Sloten, J. Goffin, B. Depreitere, The biomechanical behaviour of the bridging vein-superior sagittal sinus complex with implications for the mechanopathology of acute subdural haematoma., Journal of the mechanical behavior of biomedical materials 32C (2014) 155-165.

[32] M. Nierenberger, R. Wolfram-Gabel, S. Decock-Catrin, N. Boehm, Y. Rémond, J.-L. Kahn, S. Ahzi, Investigation of the human bridging veins structure using optical microscopy., Surgical and radiologic anatomy : SRA.

[33] J. D. Humphrey, Cardiovascular solid mechanics cells, tissues, and organs, 2002.

[34] T. Kiliç, A. Akakin, Anatomy of cerebral veins and sinuses., Frontiers of neurology and neuroscience 23 (2008) 4-15.

[35] J. Stålhand, Determination of human arterial wall parameters from clinical data., Biomechanics and modeling in mechanobiology 8 (2) (2009) 141-8.

[36] M. M. Mortazavi, M. Denning, B. Yalcin, M. M. Shoja, M. Loukas, R. S. Tubbs, The intracranial bridging veins: a comprehensive review of their history, anatomy, histology, pathology, and neurosurgical implications., Child's nervous system : ChNS : official journal of the International Society for Pediatric Neurosurgery.

[37] Z. Si, L. Luan, D. Kong, G. Zhao, H. Wang, K. Zhang, T. Yu, Q. Pang, MRI-based investigation on outflow segment of cerebral venous system under increased ICP condition., European journal of medical research 13 (3) (2008) 121-6.

[38] G. M. Treece, a. H. Gee, P. M. Mayhew, K. E. S. Poole, High resolution cortical bone thickness measurement from clinical CT data., Medical image analysis 14 (3) (2010) 276-90. 
[39] M. Visser, A. V. Muiswinkel, C. Ciuhu, G. D. Haan, Enhanced MRI Resolution for Clinical Applications, Proc. Intl. Soc. Mag. Reson. Med 14 (2006) 2396.

[40] T. Vaughan, L. DelaBarre, C. Snyder, J. Tian, C. Akgun, D. Shrivastava, W. Liu, C. Olson, G. Adriany, J. Strupp, P. Andersen, A. Gopinath, P.-F. van de Moortele, M. Garwood, K. Ugurbil, 9.4T human MRI: preliminary results., Magnetic resonance in medicine : official journal of the Society of Magnetic Resonance in Medicine / Society of Magnetic Resonance in Medicine 56 (6) (2006) 1274-82.

[41] C. Qian, I. S. Masad, J. T. Rosenberg, M. Elumalai, W. W. Brey, S. C. Grant, P. L. Gor'kov, A volume birdcage coil with an adjustable sliding tuner ring for neuroimaging in high field vertical magnets: ex and in vivo applications at 21.1T., Journal of magnetic resonance (San Diego, Calif. : 1997) 221 (2012) 110-6.

[42] H. Kiyosue, S. Tanoue, Y. Sagara, Y. Hori, M. Okahara, J. Kashiwagi, H. Nagatomi, H. Mori, The anterior medullary-anterior pontomesencephalic venous system and its bridging veins communicating to the dural sinuses: normal anatomy and drainage routes from dural arteriovenous fistulas., Neuroradiology 50 (12) (2008) 1013-23.

[43] E. Spuentrup, A. J. Wiethoff, E. C. Parsons, P. Spangenberg, C. P. Stracke, High spatial resolution magnetic resonance imaging of experimental cerebral venous thrombosis with a blood pool contrast agent., European journal of radiology 74 (3) (2010) 445-52.

[44] S. Grunt, K. Wingeier, E. Wehrli, E. Boltshauser, A. Capone, J. Fluss, D. Gubser-Mercati, P.-Y. Jeannet, E. Keller, J.-P. Marcoz, T. SchmittMechelke, P. Weber, M. Weissert, M. Steinlin, Cerebral sinus venous thrombosis in Swiss children., Developmental medicine and child neurology 52 (12) (2010) 1145-50.

[45] L. Liauw, M. a. van Buchem, a. Spilt, F. T. de Brü̈ne, R. van den Berg, J. Hermans, M. N. Wasser, MR angiography of the intracranial venous system., Radiology 214 (3) (2000) 678-82.

[46] L. Liang, Y. Korogi, T. Sugahara, M. Onomichi, Y. Shigematsu, D. Yang, M. Kitajima, Y. Hiai, M. Takahashi, Evaluation of the in- 
tracranial dural sinuses with a 3D contrast-enhanced MP-RAGE sequence: prospective comparison with 2D-TOF MR venography and digital subtraction angiography., AJNR. American journal of neuroradiology 22 (3) (2001) 481-92.

[47] P. Suetens, Fundamentals of medical imaging, 1st Edition, Cambridge University Press, 2002.

[48] C. B. Majoie, M. V. Straten, H. W. Venema, G. J. D. Heeten, Multisection CT Venography of the Dural Sinuses and Cerebral Veins by Using Matched Mask Bone Elimination (May) (2004) 787-791.

[49] D. P. Harrington, L. M. Boxt, P. D. Murray, Digital Subtraction Angiography : Overview of technical principles, American Journal of Roentgenology (139) (1982) 781-786.

[50] F. Lafitte, M. Boukobza, J. P. Guichard, C. Hoeffel, D. Reizine, O. Ille, F. Woimant, J. J. Merland, MRI and MRA for diagnosis and follow-up of cerebral venous thrombosis (CVT)., Clinical radiology 52 (9) (1997) 672-9.

[51] S. G. Wetzel, E. Kirsch, K. W. Stock, M. Kolbe, a. Kaim, E. W. Radue, Cerebral veins: comparative study of CT venography with intraarterial digital subtraction angiography., AJNR. American journal of neuroradiology 20 (2) (1999) 249-55.

[52] T. Kiliç, K. Ozduman, S. Cavdar, M. M. Ozek, M. N. Pamir, The galenic venous system: surgical anatomy and its angiographic and magnetic resonance venographic correlations., European journal of radiology 56 (2) (2005) 212-9.

[53] F. E. Hossler, J. E. Douglas, Microscopy Microanalysis Vascular Corrosion Casting : Review of Advantages and Limitations in the Application of Some Simple Quantitative Methods (2001) 253-264.

[54] T. Krucker, A. Lang, E. P. Meyer, New polyurethane-based material for vascular corrosion casting with improved physical and imaging characteristics., Microscopy research and technique 69 (2) (2006) 138-47. 
[55] J. Levesque, J. Fredrick, M. Nerem, Vascular casting: Endothelium A New Method for the Study of the Arterial, Journal of atherosclerosis 34 (77) (1979) 457-467.

[56] J. D. Bancroft, A. Stevens, Theory and practice of histological techniques, 4th Edition, Churchill Livingstone, 1996.

[57] W. Malkusch, B. Rehn, J. Bruch, Advantages of Sirius Red staining for quantitative morphometric collagen measurements in lungs., Experimental lung research 21 (1) (1995) 67-77.

[58] H. Puchtler, F. S. Waldrop, On the mechanism of Verhoeff's elastica stain: a convenient stain for myelin sheaths., Histochemistry 62 (3) (1979) 233-47.

[59] E. G. van Putten, D. Akbulut, J. Bertolotti, W. L. Vos, a. Lagendijk, a. P. Mosk, Scattering Lens Resolves Sub-100 nm Structures with Visible Light, Physical Review Letters 106 (19) (2011) 193905.

[60] S. M. Arribas, C. J. Daly, M. C. González, J. C. McGrath, Imaging the vascular wall using confocal microscopy., The Journal of physiology 584 (Pt 1) (2007) 5-9.

[61] M. K. O'Connell, S. Murthy, S. Phan, C. Xu, J. Buchanan, R. Spilker, R. L. Dalman, C. K. Zarins, W. Denk, C. A. Taylor, The threedimensional micro- and nanostructure of the aortic medial lamellar unit measured using 3d confocal and electron microscopy imaging., Matrix Biol 27 (3) (2008) 171-181.

[62] P. S. Clifford, S. R. Ella, A. J. Stupica, Z. Nourian, M. Li, L. A. Martinez-Lemus, K. A. Dora, Y. Yang, M. J. Davis, U. Pohl, G. A. Meininger, M. A. Hill, Spatial distribution and mechanical function of elastin in resistance arteries: a role in bearing longitudinal stress., Arterioscler Thromb Vasc Biol 31 (12) (2011) 2889-2896.

[63] W. Denk, K. Svoboda, Photon upmanship: why multiphoton imaging is more than a gimmick., Neuron 18 (3) (1997) 351-7.

[64] M. van Zandvoort, W. Engels, K. Douma, L. Beckers, M. Oude Egbrink, M. Daemen, D. W. Slaaf, Two-photon microscopy for imaging 
of the (atherosclerotic) vascular wall: a proof of concept study., J Vasc Res 41 (1) (2004) 54-63.

[65] R. T. A. Megens, S. Reitsma, L. Prinzen, M. G. A. oude Egbrink, W. Engels, P. J. A. Leenders, E. J. L. Brunenberg, K. D. Reesink, B. J. A. Janssen, B. M. ter Haar Romeny, D. W. Slaaf, M. A. M. J. van Zandvoort, In vivo high-resolution structural imaging of large arteries in small rodents using two-photon laser scanning microscopy., J Biomed Opt 15 (1) (2010) 011108.

[66] R. Erni, M. Rossell, C. Kisielowski, U. Dahmen, Atomic-Resolution Imaging with a Sub-50-pm Electron Probe, Physical Review Letters 102 (9) (2009) 096101.

[67] L. M. Auer, N. Ishiyama, K. C. Hodde, R. Kleinert, R. Pucher, Effect of intracranial pressure on bridging veins in rats., J Neurosurg 67 (2) (1987) 263-268.

[68] N. Famaey, J. Vander Sloten, Soft tissue modelling for applications in virtual surgery and surgical robotics., Computer methods in biomechanics and biomedical engineering 11 (4) (2008) 351-66.

[69] G. A. Holzapfel, Nonlinear Solid Mechanics: A Continuum Approach for Engineering, John Wiley \& Sons, 2000.

[70] K. L. Monson, W. Goldsmith, N. M. Barbaro, G. T. Manley, Axial Mechanical Properties of Fresh Human Cerebral Blood Vessels, Journal of Biomechanical Engineering 125 (2) (2003) 288.

[71] F. H. Silver, P. B. Snowhill, D. J. Foran, Mechanical behavior of vessel wall: a comparative study of aorta, vena cava, and carotid artery., Annals of biomedical engineering 31 (7) (2003) 793-803.

[72] G. Holzapfel, T. Gasser, M. Stadler, A structural model for the viscoelastic behavior of arterial walls: Continuum formulation and finite element analysis, European Journal of Mechanics - A/Solids 21 (3) (2002) 441-463.

[73] M. a. Zulliger, N. T. M. R. Kwak, T. Tsapikouni, N. Stergiopulos, Effects of longitudinal stretch on VSM tone and distensibility of muscular 
conduit arteries., American journal of physiology. Heart and circulatory physiology 283 (6) (2002) H2599-605.

[74] Y. C. Fung, S. C. Cowin, Biomechanics: Mechanical Properties of Living Tissues, 2nd ed., Journal of Applied Mechanics 61 (4) (1994) 1007.

[75] S. Kleiven, Predictors for traumatic brain injuries evaluated through accident reconstructions., Stapp car crash journal 51 (October) (2007) 81-114.

[76] G. A. Holzapfel, T. C. Gasser, R. A. Y. W. Ogden, A New Constitutive Framework for Arterial Wall Mechanics and a Comparative Study of Material Models, Journal of Elasticity 61 (2000) 1-48.

[77] D. P. Sokolis, Experimental investigation and constitutive modeling of the 3d histomechanical properties of vein tissue., Biomech Model Mechanobiol 12 (3) (2013) 431-451.

[78] T. C. Gasser, R. W. Ogden, G. a. Holzapfel, Hyperelastic modelling of arterial layers with distributed collagen fibre orientations., Journal of the Royal Society, Interface / the Royal Society 3 (6) (2006) 15-35.

[79] N. Famaey, G. Sommer, J. Vander Sloten, G. a. Holzapfel, Arterial clamping: finite element simulation and in vivo validation., Journal of the mechanical behavior of biomedical materials 12 (2012) 107-18.

[80] K. C. McGilvray, R. Sarkar, K. Nguyen, C. M. Puttlitz, A biomechanical analysis of venous tissue in its normal and post-phlebitic conditions., Journal of biomechanics 43 (15) (2010) 2941-7.

[81] R. Rezakhaniha, N. Stergiopulos, A structural model of the venous wall considering elastin anisotropy., Journal of biomechanical engineering 130 (3) (2008) 031017.

[82] M. a. Zulliger, P. Fridez, K. Hayashi, N. Stergiopulos, A strain energy function for arteries accounting for wall composition and structure., Journal of biomechanics 37 (7) (2004) 989-1000.

[83] M. Nierenberger, Y. Rémond, S. Ahzi, A new multiscale model for the mechanical behavior of vein walls., Journal of the mechanical behavior of biomedical materials 23 (2013) 32-43. 
[84] Y.-c. Fung, Biomechanics Mechanical properties of living tissues, 1993.

[85] D. Meaney, Biomechanics of Acute subdural Hematoma in the Subhuman Primate and Man., Ph.d. thesis, University of Pennsylvania (1991).

[86] L. Maw-Chang, Strain rate effects on tensile failure properties of the the common carotid artery and jugular veins of ferrets, Journal of biomechanics 25 (December 1991) (1992) 925-927.

[87] K. Baeck, Biomechanical modeling of head impacts: A critical analysis of finte element modeling approaches., Phd thesis, Katholieke Universiteit Leuven (2013).

[88] J. P. Xie, S. Q. Liu, R. F. Yang, Y. C. Fung, The zero-stress state of rat veins and vena cava., J Biomech Eng 113 (1) (1991) 36-41.

[89] A. Rachev, S. E. Greenwald, Residual strains in conduit arteries., J Biomech 36 (5) (2003) 661-670.

[90] D. Balzani, J. Schröder, D. Gross, Simulation of discontinuous damage incorporating residual stresses in circumferentially overstretched atherosclerotic arteries., Acta biomaterialia 2 (6) (2006) 609-18.

[91] H. Weisbecker, D. M. Pierce, P. Regitnig, G. A. Holzapfel, Layerspecific damage experiments and modeling of human thoracic and abdominal aortas with non-atherosclerotic intimal thickening., Journal of the mechanical behavior of biomedical materials 12 (2012) 93-106.

[92] N. Famaey, J. Vander Sloten, E. Kuhl, A three-constituent damage model for arterial clamping in computer-assisted surgery., Biomechanics and modeling in mechanobiology 12 (1) (2013) 123-36.

[93] G. A. Holzapfel, Determination of material models for arterial walls from uniaxial extension tests and histological structure., Journal of theoretical biology 238 (2) (2006) 290-302.

[94] S. Zeinali-Davarani, M.-J. Chow, R. Turcotte, Y. Zhang, Characterization of biaxial mechanical behavior of porcine aorta under gradual elastin degradation., Annals of biomedical engineering 41 (7) (2013) $1528-38$. 
[95] L. Tian, N. C. Chesler, In vivo and in vitro measurements of pulmonary arterial stiffness: A brief review., Pulm Circ 2 (4) (2012) 505-517.

[96] J. Liao, L. Yang, J. Grashow, M. S. Sacks, The relation between collagen fibril kinematics and mechanical properties in the mitral valve anterior leaflet., Journal of biomechanical engineering 129 (1) (2007) $78-87$.

[97] J. P. Vande Geest, M. S. Sacks, D. A. Vorp, The effects of aneurysm on the biaxial mechanical behavior of human abdominal aorta., J Biomech 39 (7) (2006) 1324-1334.

[98] A. Rachev, T. Shazly, A preliminary analysis of the data from an in vitro inflation-extension test can validate the assumption of arterial tissue elasticity., Journal of biomechanical engineering 135 (8) (2013) 84502 .

[99] J. Kim, S. Baek, Circumferential variations of mechanical behavior of the porcine thoracic aorta during the inflation test., Journal of biomechanics 44 (10) (2011) 1941-7.

[100] C. N. van den Broek, A. van der Horst, M. C. M. Rutten, F. N. van de Vosse, A generic constitutive model for the passive porcine coronary artery., Biomechanics and modeling in mechanobiology 10 (2) (2011) $249-58$.

[101] F. Service, A. Forest, S. Forest, Rail shear test for evaluating edgewise shear properties of woodbase panel products (1969).

[102] S. Lee, M. Munrot, Evaluation of in-plane shear test methods for advanced composite materials by the decision analysis technique, Composites 17 (1) (1986) 13-22.

[103] J.-S. Raul, C. Deck, R. Willinger, B. Ludes, Finite-element models of the human head and their applications in forensic practice., International journal of legal medicine 122 (5) (2008) 359-66.

[104] C. Ward, R. Thompson, The development of a detailed finite element brain model, Proceedings of the 19th Stapp Car Crash Conference, SAE Paper (1975) No. 751163. 
[105] D. Sahoo, C. Deck, R. Willinger, Finite element head model simulation and head injury prediction., Comput Methods Biomech Biomed Engin 16 Suppl 1 (2013) 198-199.

[106] K. Baeck, J. Goffin, J. Vander Sloten, An investigation into the use and limitations of different spatial integration schemes and finite element software in head impact analyses., Comput Methods Biomech Biomed Engin.

[107] S. Roth, J.-S. Raul, B. Ludes, R. Willinger, Finite element analysis of impact and shaking inflicted to a child., Int J Legal Med 121 (3) (2007) $223-228$.

[108] J.-S. Raul, S. Roth, B. Ludes, R. Willinger, Influence of the benign enlargement of the subarachnoid space on the bridging veins strain during a shaking event: a finite element study., International journal of legal medicine 122 (4) (2008) 337-40.

[109] S. Roth, J.-S. Raul, R. Willinger, Finite element modelling of paediatric head impact: global validation against experimental data., Computer methods and programs in biomedicine 99 (1) (2010) 25-33.

[110] T. Shugar, A finite element head injury model, Vol. I: Theory, development, and results., Tech. rep., U.S. Dept. Of Transportaton Report No. DOT-HS-289-3-550-IA. (1977).

[111] E. G. Takhounts, S. a. Ridella, V. Hasija, R. E. Tannous, J. Q. Campbell, D. Malone, K. Danelson, J. Stitzel, S. Rowson, S. Duma, Investigation of traumatic brain injuries using the next generation of simulated injury monitor (SIMon) finite element head model., Stapp car crash journal 52 (November) (2008) 1-31.

[112] S. Kleiven, Evaluation of head injury criteria using a finite element model validated against experiments on localized brain motion, intracerebral acceleration, and intracranial pressure, International Journal of Crashworthiness 11 (1) (2006) 65-79.

[113] M. Doorly, Investigation into head injury criteria using numerical reconstruction of real life accident cases, Ph.d. thesis, University Collega Dublin (2007). 
[114] M. S. Jazi, A. Rezaei, G. Karami, F. Azarmi, M. Ziejewski, A computational study of influence of helmet padding materials on the human brain under ballistic impacts., Computer methods in biomechanics and biomedical engineering (July 2013) (2013) 37-41.

[115] W. Yan, O. D. Pangestu, A modified human head model for the study of impact head injury., Computer methods in biomechanics and biomedical engineering 14 (12) (2011) 1049-57.

[116] C. Zhou, T. B. Khalil, A. I. King, A New Model Comparing Impact Responses of the Homogeneous and Inhomogeneous Human Brain, in: Proceedings of the 39th Stapp Car Crash Conference, November 8-10, 1995, San Diego, California, USA (sae technical paper 952714), 1995, pp. $121-37$.

[117] L. Zhang, K. H. Yang, a. I. King, Comparison of brain responses between frontal and lateral impacts by finite element modeling., Journal of neurotrauma 18 (1) (2001) 21-30.

[118] D. C. Viano, I. R. Casson, E. J. Pellman, L. Zhang, A. I. King, K. H. Yang, Concussion in Professional Football: Brain Responses by Finite Element Analysis: Part 9, Neurosurgery 57 (5) (2005) 891-916.

[119] M. Zoghi-Moghadam, A. M. Sadegh, Global/local head models to analyse cerebral blood vessel rupture leading to ASDH and SAH, Computer Methods in Biomechanics and Biomedical Engineering 12 (1) (2009) 112 .

\section{Appendix A. Basic kinematics}

Since soft biological tissues can undergo large physiological deformations, the key kinematic quantity to characterize the deformation process is the deformation gradient $\mathbf{F}$, i.e., the gradient of the deformation map $\boldsymbol{\varphi}$ with respect to the undeformed position $\boldsymbol{X}$ :

$$
\mathbf{F}=\nabla_{X} \boldsymbol{\varphi} \quad \text { and } \quad J=\operatorname{det}(\mathbf{F})
$$

Here, $J$ denotes its Jacobian $J$, which is close to one, $J \approx 1$, for nearly incompressible materials. In that case, it proves convenient to decompose 
the deformation gradient into a deviatoric part, $\overline{\mathbf{F}}$, and a volumetric part, $J^{1 / 3} \mathbf{I}$,

$$
\mathbf{F}=J^{1 / 3} \overline{\mathbf{F}}
$$

Typically, the deformation of incompressible materials is characterised in terms of the invariants of the deviatoric part $\overline{\mathbf{C}}$ of the right Cauchy-Green tensor $\mathbf{C}$, with

$$
\mathbf{C}=\mathbf{F}^{\mathrm{T}} \mathbf{F} \quad \text { and } \quad \overline{\mathbf{C}}=\overline{\mathbf{F}}^{\mathrm{T}} \overline{\mathbf{F}} .
$$

The basic deviatoric invariants $\bar{I}_{i}$ take the following explicit representation:

$$
\begin{aligned}
\bar{I}_{1} & =\operatorname{tr}(\overline{\mathbf{C}}), \\
\bar{I}_{2} & =\frac{1}{2}\left[\operatorname{tr}^{2}(\overline{\mathbf{C}})-\operatorname{tr}\left(\overline{\mathbf{C}}^{2}\right)\right], \\
\bar{I}_{3} & =\operatorname{det}(\overline{\mathbf{C}}) .
\end{aligned}
$$

The Lagrangian strain tensor is defined as a function of the right CauchyGreen tensor as:

$$
\mathbf{E}=\frac{1}{2}(\mathbf{C}-\mathbf{I})
$$

or, directly as a function of the displacement vector $\mathbf{U}$ as:

$$
\mathbf{E}=\frac{1}{2}\left(\nabla \mathbf{U}+\nabla \mathbf{U}^{T}+\nabla \mathbf{U}^{T} \nabla \mathbf{U}\right)
$$

For small displacements, the strain tensor is linearised to:

$$
\mathbf{E}_{\text {linear }}=\frac{1}{2}\left(\nabla \mathbf{U}+\nabla \mathbf{U}^{T}\right) .
$$

